\title{
String formulation of space charge forces in a deflecting bunch
}

\author{
Richard Talman \\ Laboratory of Elementary-Particle Physics, Cornell University, Ithaca, New York, USA
}

(Received 4 February 2004; published 11 October 2004)

\begin{abstract}
The force between two moving point charges, because of its inverse square law singularity, cannot be applied directly in the numerical simulation of bunch dynamics; radiative effects make this especially true for short bunches being deflected by magnets. This paper describes a formalism circumventing this restriction in which the basic ingredient is the total force on a point charge comoving with a longitudinally aligned, uniformly charged string. Bunch evolution can then be treated using direct particle-to-particle, intrabeam scattering, with no need for an intermediate, particle-in-cell, step. Electric and magnetic fields do not appear individually in the theory. Since the basic formulas are both exact (in paraxial approximation) and fully relativistic, they are applicable to beams of all particle types and all energies. But the theory is expected to be especially useful for calculating the emittance growth of the ultrashort electron bunches of current interest for energy recovery linacs and freeelectron lasers. The theory subsumes coherent synchrotron radiation and centrifugal space charge force. Renormalized, on-axis, longitudinal field components are in excellent agreement with values from Saldin et al. [DESY Report No. DESY-TESLA-FEL-96-14, 1995; Nucl. Instrum. Methods Phys. Res., Sect. A 417, 158 (1998).]
\end{abstract}

DOI: $10.1103 /$ PhysRevSTAB.7.100701

PACS numbers: 29.27.Bd, 41.60.Ap, 41.75.-i

\section{INTRODUCTION}

The electric and magnetic forces between two point charges are proportional to the inverse square of their separation distance. During the numerical simulation of the evolution of a bunch of $N$ particles, this singular behavior results in intolerably erratic behavior during close encounters. Some sort of smoothing or averaging procedure, such as particle-in-cell (PIC) code, has typically been required to overcome this problem. Here a different workaround is proposed in which the singularity is made less severe by representing point charges as longitudinally aligned, line charges or needles or "strings." These strings have zero transverse extent and length $2 L$. Until a brief discussion in a later section, it is left open whether $L$ is matched to the actual bunch length or is purely artificial (though short compared to the actual bunch length.)

The main content of this paper is closed-form expressions for the force on a point charge due to a comoving charged string. They are intended to form the basis of treatments of space charge effects as direct "intrabeam scattering" with no need for charge distribution tallying followed by field solving. Though the formalism is applicable to particles of arbitrary type and energy, the emphasis here will be on short electron bunches for which radiative effects are important. The longitudinal force component is primarily of interest for calculating coherent synchrotron radiation (CSR), the transverse component for calculating centrifugal space charge force (CSCF). Some comments concerning the history of CSCF are contained in a paper by $\mathrm{Li}$ and Derbenev [1] and there is further discussion in a paper by Geloni et al. [2]. The CSCF has been analysed by
Piwinski [3] and by Decker [4]. The effect was first introduced, calculated and named by Talman [5]. Possible restrictions to the validity of CSRF were pointed out by Lee [6] quoting Laslett [7]. Derbenev and Shiltsev [8], have also analysed CSCF.

The present paper contains formulas for all force components but the discussion will tend to emphasize longitudinal forces. This is not because they are more important-both longitudinal and transverse forces cause emittance growth, and both require renormalization. But the longitudinal component is directly related to coherent synchrotron radiation, which can be calculated by an alternative method. This provides an important test of the validity of the string formalism.

In a numerical simulation of bunch dynamics, each of the $N$ particles is to be treated as a point particle as far as its own dynamics is concerned, but as a line charge for the purpose of calculating the electromagnetic fields it generates. The forces caused by these fields can either be applied directly to each individual particle in the bunch or, for simulations in which $N$ is very large, be recorded on a three dimensional grid, from which forces on individual particles are then calculated by interpolation. In either case the basic ingredient is the force on a point charge due to a charged string.

Since this (vector) force is the result of an integration along a line charge distribution, it can be expressed as a closed-form, indefinite integral, to be evaluated at the string ends. Once formulas have been made available for these integrals, as they are in this paper, all that remains is to locate "effective" string ends, using the retarded time formalism. Though this is simple in principle, the near-cancelling coefficients, familiar from synchrotron 
radiation calculations, make this, perhaps, the hardest part of the calculation, especially in the case of bunches entering and leaving magnets. This paper describes robust numerical procedures for locating bunch ends of bunches either wholly inside or wholly outside magnets. A prescription for treating bunches entering and leaving magnets is discussed, but explicit formulas are not given.

The motivation for developing this point charge/line charge approach to space charge modeling was the desire to estimate the growth of transverse emittance due to space charge forces as a slender bunch passes through a magnetic field region. Over and above space charge effects present in straight line motion, the main longitudinal curvature effect goes by the name "coherent synchrotron radiation" and the main transverse curvature effect is called the "centrifugal space charge force." Since both effects can lead to growth of transverse emittance it is necessary to treat them together consistently. In spite of the fact that the problem is thoroughly relativistic, and therefore thoroughly nonstatic, much of the calculation can be recast as electrostatics and magnetostatics. The strategy of this paper will be, to the extent possible, to transform the problem into an elecrostatic/magnetostatic calculation, along the lines of Bassetti and Brandt [9]. Their formulas have to be altered by retarded time calculations leading to changed integration limits of effective charge distributions, and by the inclusion of "end effect" fields.

A serious conceptual problem arises, especially when calculating longitudinal effects; it is that Maxwell theory is incomplete when describing the self-force of a moving point charge. To recover self-consistency it is necessary to acknowledge the presence of ad hoc, internal, nonelectromagnetic stress forces that oppose the electromagnetic forces to cancel the overall self-force and permit the momentum of a point charge in field-free space to be constant.

There are at least two ways to calculate CSR. Relatively straightforward is to calculate energy flow in the far field radiation. Formulas are given below. Alternatively, by energy conservation, this should equal the loss of bunch energy caused by the self-force. The aforementioned divergence complicates the calculation of this self-force. There is a trick, called "renormalization" by Saldin et al. [10]. (It may be due to earlier authors such as Iogansen and Rabinovich [11] or Tamm [12].) Their trick is to subtract, from the force calculated in curved motion, the longitudinal force that would be present in straight line motion. This cancels the infinite self-force mentioned in the previous paragraph; the residual force is due entirely to the curvature. This may leave residual internal longitudinal forces but they (a) will be already present in linear motion, and hence calculable (and subtractable) as if the bunch is in free space, and (b) will cause no net acceleration of the bunch as a whole.
In Sec. VIII the renormalized, on-axis, longitudinal force is calculated by formulas from this paper and by formulas of Saldin et al., and are found to be in excellent agreement. This paper can therefore be regarded as extending the formulas of Saldin et al. both to off-axis locations and to include the transverse force components. For on-axis particles the transverse force has also been calculated by Geloni et al. [13] in a paper that also discusses transitions from outside to inside magnets. A later paper by Geloni et al. [14] discusses the off-axis problem, for vertical, but not radial, offsets. Another feature of the present paper is that it shows how the renormalization procedure (which leaves ordinary space charge in field-free regions unaccounted for) can be avoided.

The strategy in this paper is to reduce divergence problems by working only with line charges. The first thing to be done is to calculate the self-force of a longitudinally aligned, uniformly charged, moving string. Though leading-end and trailing-end forces will be found, they are only logarithmically divergent, and furthermore they are equal in magnitude but opposite in sign, thereby producing no net force on the string as a whole. This means that the renormalization mentioned in the previous paragraph, though not necessarily incorrect, is an artifact of the mathematics of intermediate stages of the calculation rather than being an inherent feature of electromagnetic theory.

Though this paper is not "string theory" as that term is currently understood, the use of the recently fancy noun "string" is not entirely inappropriate because, as in elementary-particle theory, the spurious (or at least nonelectromagnetic) self-force of a string is less divergent (only logarithmic) than is the self-force of a point charge. Though this may still leave a renormalization process necessary, the sensitivity of the procedure is greatly reduced by the gentler divergence. The paper begins with the easiest part of the calculation-the self-force of a straight charged string in field-free space.

\section{SELF-FORCE OF MOVING STRAIGHT CHARGED STRING}

A preliminary calculation will be to find the force exerted on itself by a uniformly traveling charged string. One purpose for this is to illustrate the style of calculation to be performed. The other is to prepare to handle a complication that will arise when calculating the space charge forces on a particle within a bunch traveling in an external magnetic field.

A uniformly charged string of length $2 L$, charge $q$, travels with velocity $v=\beta c \hat{\mathbf{z}}$ along the $z$ axis. Its line charge density is therefore $\lambda_{0}=q /(2 L)$. Within the charge distribution there are electric forces parallel to the axis but no magnetic forces. At position $z$ on the $z$ axis, using a formula that Jackson calls a "preliminary 
form" (of equations that both Griffiths and Jackson refer to as "Jefimenko's equations"), the electric field $\mathbf{E}(z, t)$ is given by

$$
\mathbf{E}(z, t)=-\frac{1}{4 \pi \epsilon_{0}} \int \frac{d z^{\prime}}{\left|z-z^{\prime}\right|}\left[\nabla^{\prime} \lambda+\frac{1}{c^{2}} \frac{\partial \mathbf{I}}{\partial t^{\prime}}\right]_{\mathrm{ret}},
$$

where $z^{\prime}$ is source point and $z$ is field point, and where the "ret" subscript implies that the quantity in square brackets is to be evaluated at the "retarded time" $t_{r}$ appropriate for the particular value of $z^{\prime}$, i.e., at $t_{r}=t-\left|z-z^{\prime}\right| / c$. To facilitate calculation later on it is useful to multiply the integrand by a factor $\delta\left(\tau-\left(t-\left|z-z^{\prime}\right| / c\right)\right)$. Integration over $\tau$ then undoes the damage and restores the value of the expression. Then, since both integration ranges are infinite, the order of integration can be reversed. After these operations the integral (without numerical factor) becomes

$$
\int d \tau \int \frac{d z^{\prime}}{R}\left(\nabla^{\prime} \lambda(\tau)+\frac{1}{c^{2}} \frac{\partial \mathbf{I}}{\partial \tau}(\tau)\right) \delta(\tau-(t-R / c)),
$$

where $R=\left|z-z^{\prime}\right|$. In terms of step function $U$ the charge and current densities are

$$
\begin{aligned}
\lambda\left(z^{\prime}, \tau\right) & =\lambda_{0}\left[U\left(z^{\prime}-\underline{z}^{\prime}(\tau)\right)-U\left(z^{\prime}-\bar{z}^{\prime}(\tau)\right)\right], \\
I\left(z^{\prime}, \tau\right) & =\beta c \lambda_{0}\left[U\left(z^{\prime}-\underline{z}^{\prime}(\tau)\right)-U\left(z^{\prime}-\bar{z}^{\prime}(\tau)\right)\right] .
\end{aligned}
$$

Here $\underline{z}^{\prime}(\tau)$ and $\bar{z}^{\prime}(\tau)$ are, respectively, tail and head positions of an effective charge distribution to be specified more explicitly below. The only dependencies of the charge and current distributions are through these locations; otherwise the charge density is constant, either zero or $\lambda_{0}$. The derivatives needed for Eq. (2) are

$$
\begin{aligned}
\left.\nabla^{\prime} \lambda\right|_{z} & =\lambda_{0}\left[\delta\left(z^{\prime}-\underline{z}^{\prime}(\tau)\right)-\delta\left(z^{\prime}-\bar{z}^{\prime}(\tau)\right)\right], \\
\frac{\partial I}{\partial \tau} & =-\beta^{2} c^{2} \lambda_{0}\left[\delta\left(z^{\prime}-\underline{z}^{\prime}(\tau)\right)-\delta\left(z^{\prime}-\bar{z}^{\prime}(\tau)\right)\right] .
\end{aligned}
$$

The purpose of introducing the artificial variable $\tau$ was so that these derivatives can be evaluated without reference to any subsequent specialization of the meaning of $\tau$. The time derivative is constant everywhere except at the string ends, which are always moving at speed $\beta c$. The gradient $\nabla^{\prime} \lambda$ also has transverse components; they will not be required. For an on-axis point $z$ the only component of electric field is longitudinal. Substitution into Eq. (2), using $1-\beta^{2}=1 / \gamma^{2}$, and (without loss of generality) setting $t=0$ yields

$$
\begin{aligned}
E_{z}(z, 0)= & -\frac{\lambda_{0}}{4 \pi \epsilon_{0} \gamma^{2}} \int d \tau \int \frac{d z^{\prime}}{\left|z-z^{\prime}\right|}\left[\delta\left(z^{\prime}-\underline{z}^{\prime}(\tau)\right)\right. \\
& \left.-\delta\left(z^{\prime}-\bar{z}^{\prime}(\tau)\right)\right] \delta(\tau+R / c) \\
= & -\frac{\lambda_{0}}{4 \pi \epsilon_{0} \gamma^{2}} \int \frac{d \tau}{R(\tau)}[\delta(\tau+\underline{R}(\tau) / c)-\delta(\tau \\
& +\bar{R}(\tau) / c)] .
\end{aligned}
$$

Even for this preliminary one-dimensional problem it is convenient to use unit vectors $\hat{\mathbf{z}}$ and (source point to field point unit vector) $\hat{\mathbf{r}}$ which, though equal in direction and magnitude, may be opposite in sign. The $\delta$ functions can be transformed to handle the implicit $\tau$ dependence. For example, introducing "retardation factor" $\underline{\kappa}$ defined by

$$
\underline{\kappa} \equiv \frac{\partial}{\partial \tau}\left(\tau+\frac{\left|z-\underline{z}^{\prime}(\tau)\right|}{c}\right)=1-\beta \hat{\mathbf{v}} \cdot \underline{\hat{\mathbf{r}}}
$$

the first $\delta$ function becomes

$$
\delta\left(\tau+\frac{\left|z-\underline{z}^{\prime}(\tau)\right|}{c}\right)=\frac{1}{1-\beta \hat{\mathbf{v}} \cdot \underline{\hat{\mathbf{r}}}} \delta\left(\tau-\underline{t}^{\prime}\right),
$$

where $\underline{t}^{\prime}$ is the retarded time from which the tail particle has influence at the observation point at $t=0$. For trailing and leading ends the on-axis retardation factors are

$$
\underline{\kappa}=1-\beta, \quad \bar{\kappa}=1+\beta .
$$

Combining factors, the on-axis electric field is

$$
E_{z}(z, 0)=\frac{\lambda_{0}}{4 \pi \epsilon_{0} \gamma^{2}}\left(\frac{1}{1+\beta} \frac{1}{\left|z-\bar{z}^{\prime}\right|}-\frac{1}{1-\beta} \frac{1}{\left|z-\underline{z}^{\prime}\right|}\right)
$$

The electric field at $z=0$ (now assumed to be internal to the string) is given by

$$
E_{z}(0,0)=\frac{\lambda_{0}}{4 \pi \epsilon_{0} \gamma^{2}}\left[\frac{1}{1+\beta} \frac{1}{\bar{z}^{\prime}}+\frac{1}{1-\beta} \frac{1}{z^{\prime}}\right] .
$$

(In simplifying this formula the "obvious" relations $\underline{z}^{\prime}<$ 0 and $0<\bar{z}^{\prime}$ have been assumed. For points exactly on the line of charge these are correct. But, anticipating later discussion, for a point displaced transversely, say by a finite height $y$, no matter how small, one or the other of these inequalities may be incorrect.) Next the retarded times and positions will be determined.

Referring to Fig. 1, a test point within the string, displaced by $z_{t}$ from string center, arrives at point $P\left(z^{\prime}=\right.$ 0 ) at time $t$. The head particle emits a signal at time $\bar{t}_{r}$ as it passes the point $z^{\prime}=\bar{z}^{\prime}$. For this signal's arrival to coincide with the test point's arrival at $P$ requires

$$
\bar{t}_{r}+\frac{\bar{z}^{\prime}}{c}=t, \quad \underline{t}_{r}-\frac{\underline{z}^{\prime}}{c}=t,
$$

where the tail equation has also been written. [The times $t$ and $t_{r}$ in Eqs. (11) are "laboratory time," as measured by clocks stationary in the laboratory. Since the events are at different locations it is necessary to use two previously synchronized clocks, one at each event. This same comment applies to all times appearing in this paper and there will never be any introduction of moving coordinate frames, nor Lorentz transformation.] Note that both $\underline{t}_{r}$ and $\bar{t}_{r}$ precede $t$.

Let us set $t=0$; that is, the test point arrives at point $P$ at $t=0$. At that instant, relative to test point, the head particle is at $z=L-z_{t}$. The equations of motion of head (and similarly for tail) are therefore 


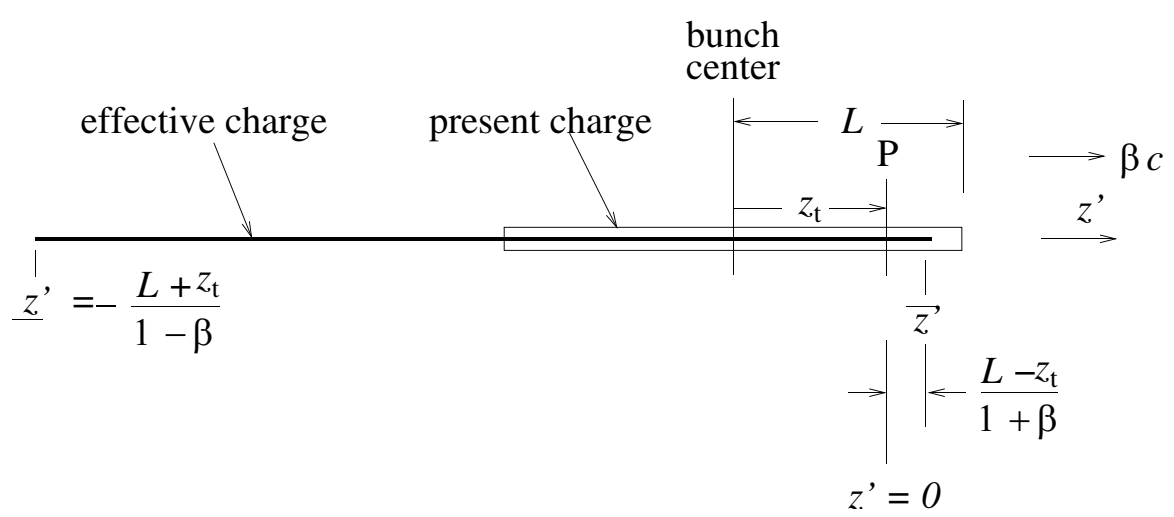

FIG. 1. Definition of coordinates. Snapshot of moving string (the open rectangle) at the "present" time $t=0$. The solid line indicates the electrostatic configuration temporarily equivalent to the "true" electrodynamic system of a moving charged string; there are charges in just those locations that, at some time $t<0$, contribute to the electric field at test point $P$ at $t=0$. In this figure that point is labeled $P$ and is instantaneously located at $z=0$.

$$
\bar{z}^{\prime}=\left(L-z_{t}\right)+\beta c \bar{t}_{r}, \quad \underline{z}^{\prime}=\left(-L-z_{t}\right)+\beta c \underline{t}_{r}
$$

Eliminating $\bar{z}^{\prime}$ and $\underline{z}^{\prime}$ from these equations yields

$$
c \bar{t}_{r}=\frac{-L+z_{t}}{1+\beta}, \quad c \underline{t}_{r}=-\frac{L+z_{t}}{1-\beta} .
$$

(Since both values of $t_{r}$ are necessarily negative it would be more apt to refer to $t_{r}$ as "earlier time" rather than as retarded time, which is the customary terminology.) Solving for $\bar{z}^{\prime}$ and $\underline{z}^{\prime}$ yields

$$
\bar{z}^{\prime}=\frac{L-z_{t}}{1+\beta}, \quad \underline{z}^{\prime}=\frac{-L-z_{t}}{1-\beta}
$$

These points delimit the effective charge distribution.

Returning to the self-force calculation, the end coordinates can be substituted into Eq. (10) to produce

$$
E_{z}\left(z_{t}\right)=\frac{\lambda_{0}}{4 \pi \epsilon_{0} \gamma^{2}}\left(\frac{1}{L-z_{t}}-\frac{1}{L+z_{t}}\right)
$$

Another way of calculating this electric field is to start from the Heaviside, Poincaré, Schott formula for the electric field accompanying a uniformly moving point charge $\lambda_{0}|d \zeta|$ as viewed at transverse position $y$ and longitudinal position $\zeta$;

$$
d \mathbf{E}(\mathbf{r}, t)=\frac{\lambda_{0}|d \zeta|}{4 \pi \epsilon_{0} \gamma^{2}} \frac{1}{\left(1-\beta^{2} \sin ^{2} \theta\right)^{3 / 2}} \frac{\hat{\mathbf{r}}}{\zeta^{2}+y^{2}},
$$

where $\theta$ is the angle between the vector from source point to field point and the trajectory direction. When expressed in this way, in terms of $\theta$, the formula is uniformly valid both in front of and behind the moving charge, and the longitudinal integration yields for the longitudinal electric field

$$
\begin{aligned}
E_{z}(\mathbf{r}, t) & =\frac{\lambda_{0}}{4 \pi \epsilon_{0} \gamma^{2} y} \int_{\underline{\theta}}^{\bar{\theta}} \frac{d(\sin \theta)}{\left(1-\beta^{2} \sin ^{2} \theta\right)^{3 / 2}} \\
& =\frac{\lambda_{0}}{4 \pi \epsilon_{0} \gamma^{2}}\left[\frac{\sin \theta / y}{\left(1-\beta^{2} \sin ^{2} \theta\right)^{1 / 2}}\right]_{\underline{\theta}}^{\bar{\theta}}
\end{aligned}
$$

On axis, this agrees with Eq. (15). Another check is contained in a paper by Jefimenko [15] which yields the same result.

Later we will take yet another (only slightly different) approach, starting from the final Jefimenko equation for $\mathbf{E}(\mathbf{r}, t)$;

$$
\frac{1}{4 \pi \epsilon_{0}} \int\left[\frac{\lambda\left(z^{\prime}, t^{\prime}\right) \hat{\mathbf{r}}}{\left(z-z^{\prime}\right)^{2}}+\frac{\left(\partial \lambda / \partial t^{\prime}\right) \hat{\mathbf{r}} / c-\left(\partial I / \partial t^{\prime}\right) \hat{\mathbf{z}} / c^{2}}{\left|z-z^{\prime}\right|}\right] d z^{\prime}
$$

Though this formula has great heuristic virtue, in order for it to be valid, as Jackson emphasizes, extreme care is needed in interpreting the partial derivative symbols. In particular, as well as the square bracket being evaluated at retarded time $t^{\prime}$, the observation time $t$ is to be held constant as the partials are evaluated. This is harder than it sounds.

The first two terms of Eq. (18) result from a perhaps subtle interpretation and a differentiation by parts, but the third term has simply been copied from Eq. (1); indicating it by superscript (3), its value has already been calculated to be

$$
E_{z}^{(3)}\left(z_{t}\right)=-\frac{\lambda_{0} \beta^{2}}{4 \pi \epsilon_{0}}\left(\frac{1}{1+\beta} \frac{1}{\bar{z}^{\prime}}+\frac{1}{1-\beta} \frac{1}{\underline{z}^{\prime}}\right) .
$$

The first term of Eq. (18) can be interpreted as Coulomb's law applied to the effective linear charge distribution running from $z^{\prime}$ to $\bar{z}^{\prime}$-in fact this is the motivation underlying the Jefimenko equation. Again referring to Fig. 1, the electric field at a point $z_{t}$ closer to the front than to the back of the line charge can be calculated at time $t=0$. The result, after setting $z=0$, is 


$$
E_{z}^{(1)}\left(z_{t}\right)=\frac{\lambda_{0}}{4 \pi \epsilon_{0}} \int_{\underline{z}^{\prime}}^{-\bar{z}^{\prime}} \frac{d z^{\prime}}{z^{\prime 2}}=\frac{\lambda_{0}}{4 \pi \epsilon_{0}}\left[\frac{1}{\bar{z}^{\prime}}+\frac{1}{\underline{z}^{\prime}}\right]
$$

In this integral, coming from the first term of Eq. (18), $z$ has been set to zero in order to find the electric field at $z_{t}$. The (divergent) contributions from intervals adjacent and symmetric relative to the test point have cancelled by symmetry. This comment is only valid for $-\bar{z}>\underline{z}$, but repeating the calculation for points closer to the tail gives the same result.

All that remains is to evaluate the second term of Eq. (18). For a particular feature, say the trailing end for definiteness, consider the evaluation of $\partial U\left(z^{\prime}-\right.$ $\left.z^{\prime}\right) / \partial t^{\prime}$. The implicit dependence of argument on $t^{\prime}$ and the need to hold $t$ fixed strains the partial derivative notation enough to make the derivative perhaps ambiguous. Proceeding cautiously, the derivative is

$$
\frac{\partial}{\partial t^{\prime}} U\left(z^{\prime}-\underline{z}^{\prime}\left(t^{\prime}\right)\right)=\delta\left(z^{\prime}-\underline{z}^{\prime}\left(t^{\prime}\right)\right)(-\beta c)
$$

which makes the trailing-edge term be

$$
-\frac{\lambda_{0}}{4 \pi \epsilon_{0}} \beta \int d z^{\prime} \frac{\hat{\mathbf{r}}}{\left|z-z^{\prime}\right|}\left[\delta\left(z^{\prime}-\underline{z}^{\prime}\left(t^{\prime}\right)\right)\right] .
$$

Performing the integral as before and combining factors, we obtain, for the second term of Eq. (18),

$\bar{E}_{z}^{(2)}\left(z_{t}\right)=\frac{\lambda_{0}}{4 \pi \epsilon_{0}} \frac{\beta}{1+\beta} \frac{-1}{\left|z-\bar{z}^{\prime}\right|_{z=0}}=\frac{\lambda_{0}}{4 \pi \epsilon_{0}} \frac{-\beta}{1+\beta} \frac{1}{\bar{z}^{\prime}}$,
$\underline{E}_{z}^{(2)}\left(z_{t}\right)=-\frac{\lambda_{0}}{4 \pi \epsilon_{0}} \frac{\beta}{1-\beta} \frac{1}{\left|z-\underline{z}^{\prime}\right|_{z=0}}=\frac{\lambda_{0}}{4 \pi \epsilon_{0}} \frac{\beta}{1-\beta} \frac{1}{\underline{z}^{\prime}}$.

Summing the fields given by Eqs. (19), (20), and (23), the result agrees with Eq. (10). This confirms the validity of the recent, possibly dubious, differentiation.

The total force acting on the string is obtained by integrating Eq. (15) over $z_{t}$;
$\int_{-L}^{L} E_{z}\left(z_{t}\right) \lambda_{0} d z_{t}=\frac{q^{2} /\left(4 L^{2}\right)}{4 \pi \epsilon_{0} \gamma^{2}} \int_{-L}^{L} d z_{t}\left[\frac{1}{L-z_{t}}-\frac{1}{L+z_{t}}\right]$

In spite of the logarithmic divergence at each end, their sum vanishes - a very satisfactory result. But the string tension is infinite and when $L$ is allowed to approach zero, the total force becomes ambiguous. This is presumably a manifestation of the fact that charges of zero extent cannot be consistently incorporated into electromagnetism without introducing further effects such as nonelectromagnetic internal stresses. Unlike point charges, where the divergence is worse, the present end divergences are only logarithmic (and suppressed by a factor of $1 / \gamma^{2}$ at that) and for straight line motion they cancel. It is the only weak divergence of the self-force that makes me feel justified in using the term string, since it is presumably very similar considerations that simplify the renormalizability of string theory. For motion of the string along a curved path in a magnetic field the end cancellation may no longer be perfect. What to do about this possibility will be discussed next.

\section{SELF-FORCE OF MOVING STRAIGHT CHARGED RIBBON}

A way of regularizing the self-force is to give the string of charge some transverse size, say a height $d$, as shown in Fig. 2, and call it a moving ribbon. To calculate the selfforce let us first find the force of a subribbon at $y_{2}$ due to a subribbon at $y_{1}$. These ribbons have transverse separation $y=y_{2}-y_{1}$.

A new feature that has to be appreciated is that the transverse displacement $y$ has a big effect on the longitudinal force. One aspect of this is indicated qualitatively in the caption of the figure. Another aspect (for relativistic motion) concerns signal propagation between two initially side-by-side particles, one on subribbon (1), the other on (2). The longitudinal distance side-by-side particles have traveled when the signal from one arrives at

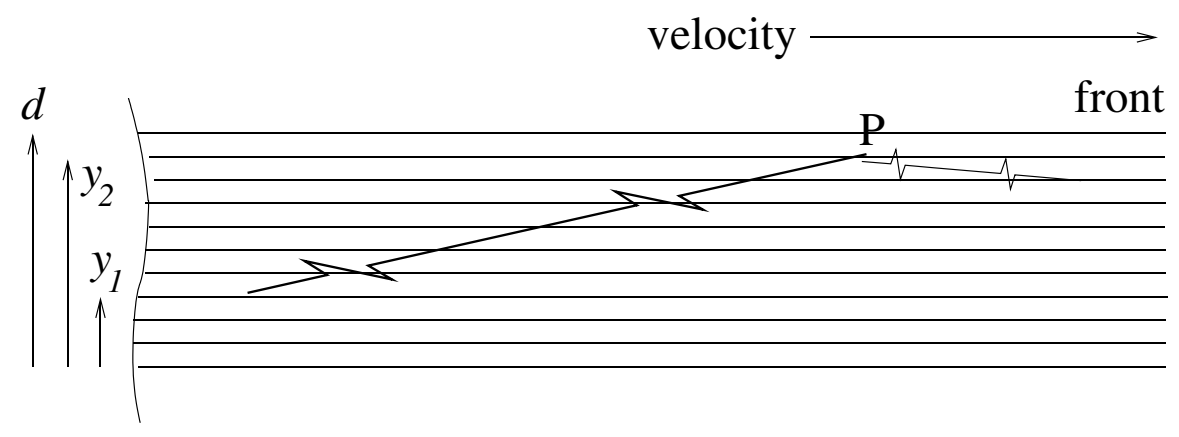

FIG. 2. Configuration used to calculate the self-force of a moving ribbon of charge. For subribbons transversely close, the point $P$ is largely influenced by points further forward in the bunch that take advantage of their earlier arrival to "send signals back." Subribbons transversely farther away from the point $P$ can be influential only by sending a signal from far enough back for the slight $c>v$ signal speed advantage to make up for the extra signal path length. 


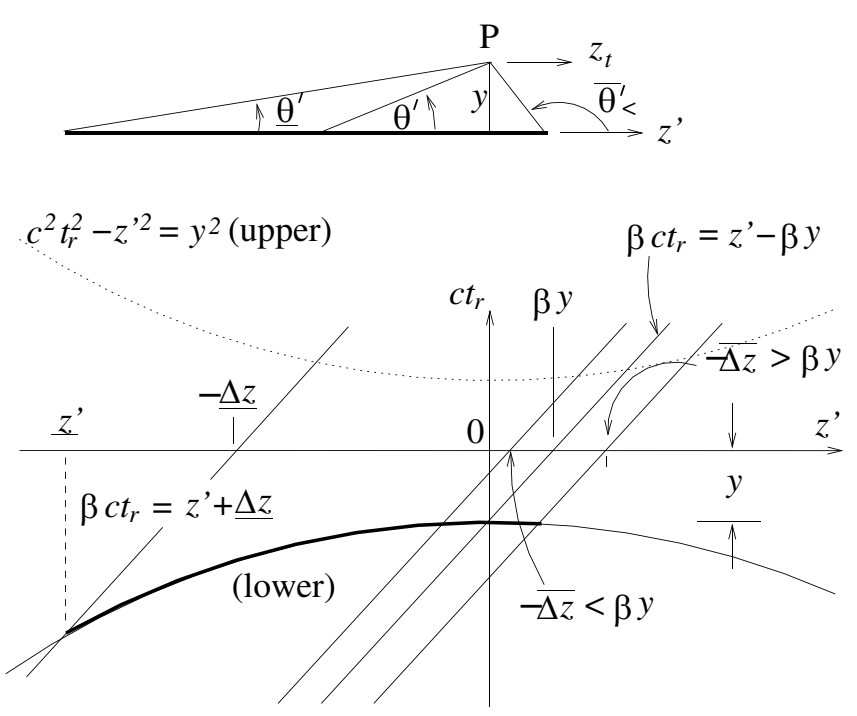

FIG. 3. Figure illustrating the $y \neq 0$ retarded time calculation. The upper figure shows the effective electrostatic configuration. In the lower figure the lower hyperbolic branch contains points from which emitted signals arrive at observation point $P$ at $t=0$. Straight lines are world lines of point charges in the line charge distribution. Heavy lines in both figures correspond to the effective charge distribution. Depending on $y$, the head may be longitudinally in front of, or behind, the test point.

the other is given by

$$
\delta_{12} \approx \gamma|y| \text {. }
$$

Even for small $y$ this can be large, even far larger than the length of a slim bunch.

The effect of nonzero $y$ on the retarded time calculation is illustrated by Fig. 3. The lower branch of hyperbola

$$
c^{2} t_{r}^{2}-z^{\prime 2}=y^{2}
$$

contains points with time and position coordinates $\left(t_{r}, z^{\prime}\right)$ on the string axis from which emitted signals arrive at observation point at $t=0$. (The upper branch would apply to an irrelevant "advanced time" calculation.) Consider the charge at position $z_{s}$ relative to string center; relative to this charge the test point $z_{t}$ is displaced by $\Delta z=z_{t}-z_{s}$. With time and space origins adjusted to vanish instantaneously at the test point, the world line of the source point is

$$
c t_{r}=\frac{z^{\prime}+\Delta z}{\beta} .
$$

The intersection of line and hyperbola gives the $\left(c t_{r}, z^{\prime}\right)$ coordinates of the particular source charge influencing the particular test charge at $t=0$. In particular, the straight lines passing through the string extremes define the effective electrostatic line charge. Contrary to a validonly-for- $y=0$ assumption mentioned just below Eq. (11), one sees that the most forward source point can be behind (i.e., at less positive longitudinal position) than the test

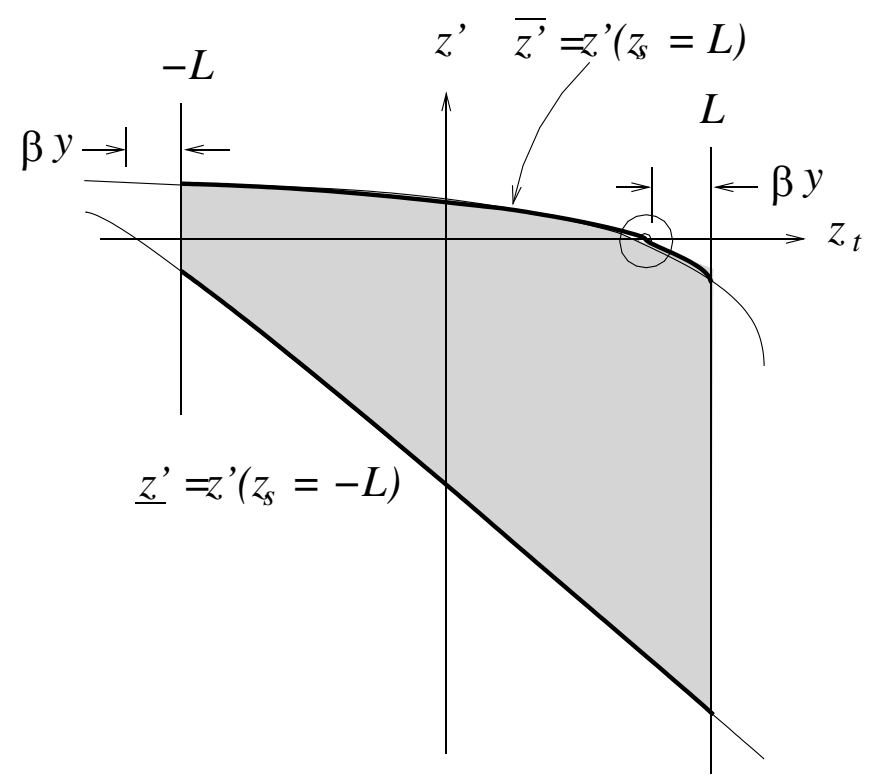

FIG. 4. The region of effective charge contributing to the total bunch self-force. At given test point $z_{t}$ the uniform charge distribution runs from $\underline{z}^{\prime}=z^{\prime}\left(z_{s}=-L, y\right)$ to $\bar{z}^{\prime}=z^{\prime}\left(z_{s}=\right.$ $-L, y)$. The open circle indicate a troublesome point at which there is a "one-sided" singularity in the integral over $z_{t}$ for $y \rightarrow$ 0 .

point. The dividing condition, for effective charge front to coincide longitudinally with test point, is

$$
\bar{z}^{\prime}=0 \quad \text { or } \quad \Delta z=-\beta y \quad \text { or } \quad z_{s}=z_{t}+\beta y .
$$

These conditions are illustrated in Fig. 4. For charges in the interval of length $\beta y$ at the front of the actual bunch, even apart from any dependence of electric field on $y$, this invalidates some relative-position assumptions made while obtaining the $y=0$ field.

A workaround for this problem is suggested by the upper part of Fig. 3. It is to express the range of the effective charge distribution by angular coordinate $\theta^{\prime}$ instead of by rectilinear coordinate $z^{\prime}$. For $y>0$ the entire range of $\theta^{\prime}$ is well behaved. Setting up the integral analogous to Eq. (20) and performing the integration, one finds that the longitudinal electric field at $P$ due to the effective line charge (end contributions temporarily neglected) is given by

$$
d E_{z}\left(z_{t}, y\right)=\frac{\lambda_{0}\left(d y_{1} / d\right)}{4 \pi \epsilon_{0} y}\left[\sin \bar{\theta}^{\prime}-\sin \underline{\theta}^{\prime}\right] .
$$

For consistency, having admitted finite $y$, it is necessary to consider magnetic fields as well. With $\mathbf{r}$ being vector from source point to field point, so $r^{2}=z^{\prime 2}+y^{2}$, the complete Jefimenko equations are

$$
\mathbf{E}(P, t)=\frac{1}{4 \pi \epsilon_{0}} \int\left[\frac{\lambda\left(z^{\prime}, t_{r}\right)}{r^{2}} \hat{\mathbf{r}}+\frac{\dot{\lambda}\left(z^{\prime}, t_{r}\right)}{c r} \hat{\mathbf{r}}-\frac{\dot{\mathbf{I}}\left(z^{\prime}, t_{r}\right)}{c^{2} r}\right] d z^{\prime},
$$




$$
\mathbf{B}(P, t)=\frac{\mu_{0}}{4 \pi} \int\left[\frac{\mathbf{I}\left(z^{\prime}, t_{r}\right)}{r^{2}}+\frac{\dot{\mathbf{I}}\left(z^{\prime}, t_{r}\right)}{c r}\right] \times \hat{\mathbf{r}} d z^{\prime} .
$$

Here, following Griffiths's somewhat casual notation, partial derivatives with respect to "time" are indicated by overhead dots. These derivatives assume $z^{\prime}$ is held fixed, and the resulting quantity evaluated at the retarded time appropriate for that value of $z^{\prime}$. In other words the overhead dots have the same meaning as the partial derivatives in Eq. (18). (This is not at all the same as the convention invented by Newton and customary in mechanics.) We are mainly interested in the total force, say on charge $e$, which is given by

$$
\frac{\mathbf{F}(P)}{e}=\mathbf{E}(P)+\beta c \hat{\mathbf{z}} \times \mathbf{B}(P) .
$$

Substituting from Eqs. (30) and (31) and reducing the result produces body contribution

$$
\begin{aligned}
d \mathbf{F}^{\text {body }} / e & =\frac{\lambda_{0} d y_{1} / d}{4 \pi \epsilon_{0} y}\left[\frac{\sin \theta^{\prime}}{\gamma^{2}} \hat{\mathbf{y}}+\cos \theta^{\prime} \hat{\mathbf{z}}\right] d z^{\prime} \\
& =\frac{\lambda_{0} d y_{1} / d}{4 \pi \epsilon_{0} y}\left[-\frac{\cos \theta^{\prime}}{\gamma^{2}} \hat{\mathbf{y}}+\sin \theta^{\prime} \hat{\mathbf{z}}\right]_{\underline{\theta}^{\prime}}^{\bar{\theta}^{\prime}}
\end{aligned}
$$

and end contribution

$$
\begin{aligned}
d \mathbf{F}^{\mathrm{ends}} / e= & \frac{d y_{1} / d}{4 \pi \epsilon_{0}} \int \frac{\dot{\lambda}}{c r}\left[\frac{\sin \theta^{\prime}}{\gamma^{2}} \hat{\mathbf{y}}+\left(\cos \theta^{\prime}-\beta\right) \hat{\mathbf{z}}\right] d z^{\prime} \\
= & \frac{\lambda_{0} d y_{1} / d}{4 \pi \epsilon_{0}}\left[\frac { \beta } { 1 - \beta \operatorname { c o s } \theta ^ { \prime } } \left(\frac{\sin \theta^{\prime}}{\gamma^{2} r^{\prime}} \hat{\mathbf{y}}\right.\right. \\
& \left.\left.+\frac{\cos \theta^{\prime}-\beta}{r^{\prime}} \hat{\mathbf{z}}\right)\right]_{\underline{\theta}^{\prime}, \underline{r}^{\prime}}^{\bar{\theta}^{\prime}, \bar{r}^{\prime}}
\end{aligned}
$$

The end corrections have been calculated as in Eq. (22), using $\kappa=1-\beta \cos \theta^{\prime}$. Combining body and end contributions, for $z=0$ the longitudinal force is

$$
\frac{d F_{z}}{e}=\frac{\lambda_{0} d y_{1} / d}{4 \pi \epsilon_{0} \gamma^{2}}\left[\frac{1}{r^{\prime}-\beta r^{\prime} \cos \theta^{\prime}}\right]_{\underline{\theta}^{\prime}, \underline{r}^{\prime}}^{\bar{\theta}^{\prime}, \bar{r}^{\prime}} .
$$

Since no approximations have been made, nor assumptions about the relative position of source points and field point, this formula is valid everywhere in space. But it is inconveniently expressed in terms of retarded coordinates. It will be convenient to convert to coordinates related to the actual, i.e., present, position of the string. For this calculation Fig. 5 is useful. The master relationships, as shown in the figure, are

$$
-\underline{z}^{\prime}-\left(L+z_{t}\right)=\beta \underline{r}^{\prime} \quad \text { and } \quad\left(L-z_{t}\right)-\bar{z}^{\prime}=\beta \bar{r}^{\prime} .
$$

The first of these equations shows that the trailing edge travels from its retarded position to its present position at speed $v$ while a signal travels from the retarded position to the observation point at speed $c$. Also one has

$$
\underline{r}^{\prime} \cos \underline{\theta}^{\prime}=-\underline{z}^{\prime} \quad \text { and } \quad \bar{r}^{\prime} \cos \bar{\theta}^{\prime}=-\bar{z}^{\prime} .
$$

Equations (36) and (37) remain valid even if the test point lies longitudinally outside the true charge distribution. Using the figure, and following a manipulation suggested by Jefimenko, the needed, trailing-edge, denominator factor $\underline{r}^{\prime}-\beta \underline{r}^{\prime} \cos \underline{\theta}^{\prime}$ can be expressed as

$$
\sqrt{\underline{r}^{\prime 2}+2 \beta \underline{r}^{\prime} \underline{z}^{\prime}+\beta^{2} \underline{z}^{\prime 2}+\underline{z}^{\prime 2}-\underline{z}^{\prime 2}+\beta^{2} \underline{r}^{\prime 2}-\beta^{2} \underline{r}^{\prime 2}},
$$

with terms having been judiciously added and subtracted. The terms can be regrouped using $\underline{r}^{\prime 2}-z^{\prime 2}=y^{2}$ and [from Eq. (36)] $\left(L+z_{t}\right)^{2}=\underline{z}^{\prime 2}+2 \beta \underline{z}^{\prime} \underline{r}^{\prime}+\bar{\beta}^{2} \underline{r}^{\prime 2}$. The result is

$$
\underline{r}^{\prime}-\beta \underline{r}^{\prime} \cos \underline{\theta}^{\prime}=\sqrt{\left(L+z_{t}\right)^{2}+y^{2} / \gamma^{2}} .
$$

This result can be generalized to

$$
r^{\prime}-\beta r^{\prime} \cos \theta^{\prime}=\sqrt{\Delta z^{2}+y^{2} / \gamma^{2}},
$$

where $\Delta z=z_{t}-z_{s}$ is the relative longitudinal coordinate introduced previously. Using this result, the differential longitudinal force is

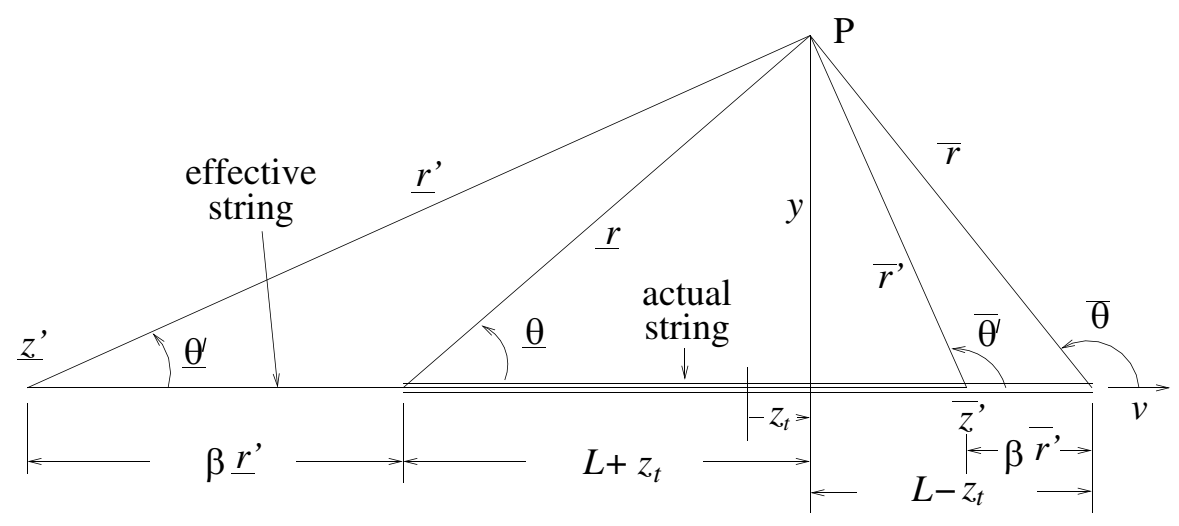

FIG. 5. Geometric relationships between present string coordinates and retarded string coordinates. As drawn $\bar{z}^{\prime}$ is positive (i.e., to the right of $P$ ) but, for $y$ as large as shown, $\bar{z}^{\prime}$ would be negative for highly relativistic motion. 


$$
\begin{aligned}
\frac{d F_{z}}{e}= & \frac{\lambda_{0} d y_{1} / d}{4 \pi \epsilon_{0} \gamma^{2}}\left[\frac{1}{\sqrt{\left(L-z_{t}\right)^{2}+y^{2} / \gamma^{2}}}\right. \\
& \left.-\frac{1}{\sqrt{\left(L+z_{t}\right)^{2}+y^{2} / \gamma^{2}}}\right] .
\end{aligned}
$$

The total longitudinal force on subribbon (2) due to subribbon (1) is given by

$$
d^{2} F_{z}(y)=\frac{\lambda_{0} d y_{2}}{d} \int_{-L}^{L} d z_{t} \frac{d F_{z}}{e} .
$$

This integral vanishes because the integrand is an odd function of $z_{t}$. If one insists on calculating a fractional force, say on the front half of the ribbon, after integration over $z_{t}, y_{1}$, and $y_{2}$, the resulting force will be convergent; the divergent force has been moderated to produce only a term proportional to $\ln d$ which is only weakly divergent as $d \rightarrow 0$.

Since the magnetic field contributes no longitudinal force, Eq. (42) should also be equivalent to Eq. (17). To confirm this observe that

$$
\frac{\sin \theta / y}{\sqrt{1-\beta^{2} \sin ^{2} \theta}}=\frac{1}{\sqrt{y^{2} / \tan ^{2} \theta+y^{2} / \gamma^{2}}}=\frac{1}{\sqrt{\Delta z^{2}+y^{2} / \gamma^{2}}},
$$

where $\theta$ is the angle from present source point to present test point.

The transverse force can be calculated similarly. Summing body and end contributions, for $z=0$, analogous to Eq. (35), the $y$ component of force is

$$
\frac{d F_{y}}{e}=\frac{\lambda_{0} d y_{1} / d}{4 \pi \epsilon_{0} \gamma^{2}}\left[-\frac{\cos \theta^{\prime}}{y}+\frac{\beta \sin \theta^{\prime}}{r^{\prime}-\beta r^{\prime} \cos \theta^{\prime}}\right]_{\underline{\theta}^{\prime}, \underline{r}^{\prime}}^{\bar{\theta}^{\prime}, \bar{r}^{\prime}} .
$$

Manipulations like those used for the longitudinal force lead to the identity

$$
-\frac{\cos \theta^{\prime}}{y}+\frac{\beta \sin \theta^{\prime}}{r^{\prime}-\beta r^{\prime} \cos \theta^{\prime}}=\frac{-\Delta z / y}{\sqrt{\Delta z^{2}+y^{2} / \gamma^{2}}} .
$$

The square bracket expression in Eq. (44) therefore reduces to

$$
-\frac{1}{y}\left[\frac{L-z_{t}}{\sqrt{\left(L-z_{t}\right)^{2}+y^{2} / \gamma^{2}}}-\frac{L+z_{t}}{\sqrt{\left(L+z_{t}\right)^{2}+y^{2} / \gamma^{2}}}\right] .
$$

The structures of Eq. (41) and (46) are similar and noteworthy. Naturally, being the result of integrations, they are differences of indefinite integrals evaluated at string head and tail. But the indefinite integral, especially for $d F_{z}$, is a function only of a "generalized" present distance $\sqrt{\left(L \pm z_{t}\right)^{2}+y^{2} / \gamma^{2}}$ from field point $P$ to string end. There is a kind of "inverse distance force law," attractive toward the leading end (for test particles within the string), repulsion from the trailing end. This force is far from being directed along the line from one charge to the other, however, and $d F_{z}$ does not reverse sign as the test point is moved longitudinally past the head of the string. One expects the transverse force to depend primarily on $y$. Equation (46) confirms this by showing a leading $1 / y$ dependence. But this factor is "modulated" by a difference of terms depending only on distances to the string ends, or rather on the ratios of actual distance divided by generalized distance. Except for $z_{t}$ very close to either end, cancellation of the terms within the square brackets suppresses the $1 / y$ singularity at $y=0$.

It is consistent to pretend that the total force is due entirely to two "sources," one at the head, one at the tail, with source "strength" given by the value of the indefinite integral. In this picture there are no force contributions from the charge in the interior of the string - not even proportional to $1 / \gamma^{2}$. This suggests a simple numerical procedure for calculating the effects of space charge forces on a beam traveling in a field-free region. With the bunch represented as a superposition of longitudinal strings, the force on any single particle depends only on its relation to the ends of the other strings. Because of the inverse dependence on $y$ in Eq. (46) the end forces are not, however, directed radially along the line joining end points and field point. These forces are intended to form the basis for treating space charge effects as direct intrabeam scattering forces.

Accelerator beams are typically considerably longer than they are broad or high. This large aspect ratio gets effectively increased by the further factor of $\gamma$ by which $y$ is divided in the "generalized distance" appearing in the denominators. This provides further heuristic explanation of the surprisingly small space charge effects in accelerator beams.

\section{CURVE END POINT DETERMINATION}

Finally we start on the real problem, which is to calculate the space charge force from a longitudinally aligned, curved (due to its presence in a magnetic field) string of charge of length $2 L$, uniform charge density $\lambda_{0}$, acting on a comoving "test charge" as it passes some nominal lattice point $P$. The plan is, to the extent possible, to recast this electrodynamic problem into an "equivalent" electrostatic/magnetostatic problem, following the pattern of the previous sections.

As far as possible notation will be carried over from the preceding discussion. In particular, retarded locations will be indicated by symbols with primes. (With retarded locations being regarded as source points, this is quite intuitive. The only important exception is that $\mathbf{r}^{\prime}$ is to be the vector directed from retarded source point to test point $P$, as in Fig. 5.)

Consider the distribution of charges illustrated in Fig. 6. An arc of charge, linear charge density $\lambda_{0}$, flows along the arc of a circle of radius $R$ with speed $\beta c$. The single test charge (charge $=e$, longitudinal position $s_{t}$, transverse coordinates $x, y$, all relative to string center) is 


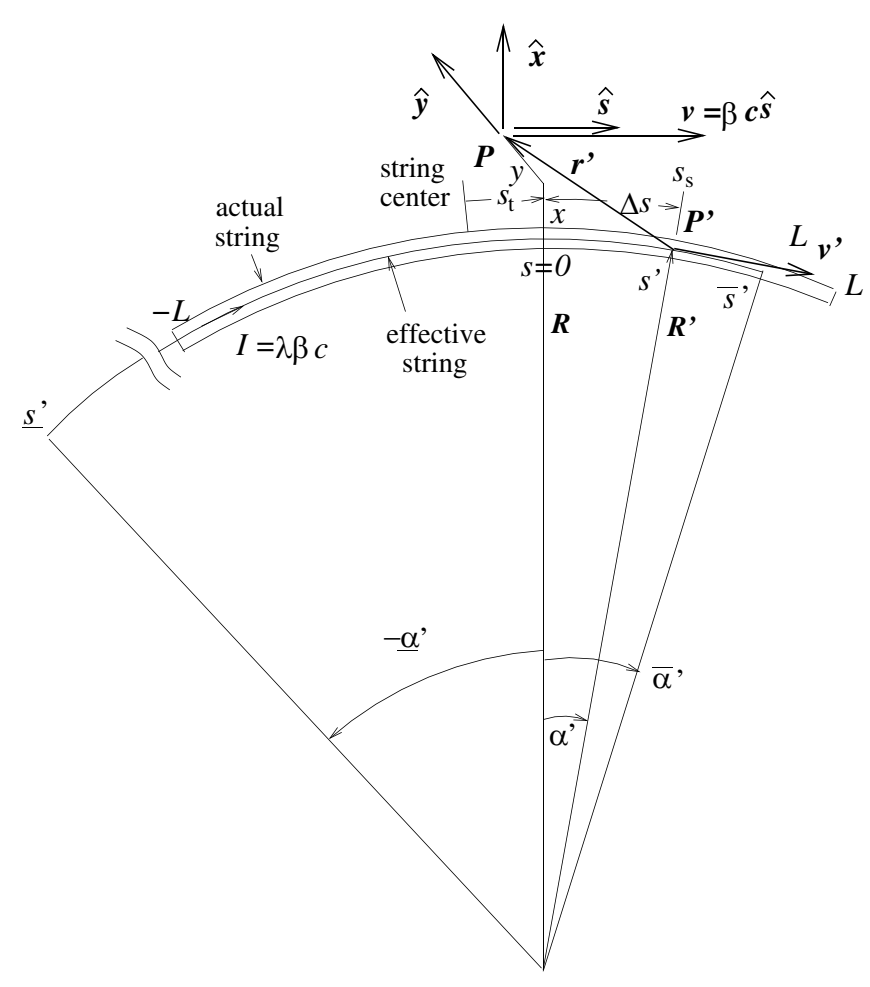

FIG. 6. Definition of coordinates. A snapshot of the present, actual, charge distribution is shown, and superimposed on it is an electrostatic/magnetostatic configuration equivalent to the true electrodynamic system of a moving charged string. There are moving charges in just those locations of charges in the true system that, at some earlier time $t<0$, contribute to fields at $P$ at $t=0$.

traveling with velocity $\beta c \hat{\mathbf{s}}$ parallel to the charged string. After finding the force, due to incremental length $R d \alpha^{\prime}$ at source point $P^{\prime}$, we will find the total force on the test charge at $P$ by integrating along the arc.

Choosing the origin of time at the instant the test charge passes point $P$ in the storage ring, the test charge's equation of motion is

$$
s_{t}=\beta c t .
$$

Let the longitudinal coordinate of the test particle relative to some particular source particle be

$$
\Delta s \equiv s_{t}-s_{s}
$$

[For example, for the source particle being at the head of the string, $\Delta s=s_{t}-L \equiv \overline{\Delta s}$, which is negative (or zero).] The equation of motion of the source particle is therefore

$$
s^{\prime}=-\Delta s+\beta c t_{r}
$$

This corresponds to Eqs. (12) but differs in that the equation describes a general source particle rather than the head or tail. Since it will be convenient to use angular coordinates rather than arc length, define

$$
\alpha^{\prime}=\frac{s^{\prime}}{R}
$$

The equation of motion of the source particle can therefore also be expressed as

$$
R \alpha^{\prime}=\beta c t_{r}-\Delta s
$$

Charge and current densities are to be evaluated at time $t_{r}$, which is related to observation time $t$ by

$$
t=t_{r}+\frac{r^{\prime}}{c}
$$

where $\mathbf{r}^{\prime}$ is the vector from source point to field point. The length $r^{\prime}$ of this vector is given by

$$
r^{\prime}=\sqrt{R^{2}+(R+x)^{2}-2 R(R+x) \cos \alpha^{\prime}+y^{2}} .
$$

Setting $t=0$ in Eq. (52), and combining it with Eqs. (51) and (53), the condition for time $t_{r}$ at which charge at $P^{\prime}$ influences fields at $P$ at $t=0$, is

$$
\frac{R \alpha^{\prime}+\Delta s}{\beta}=-\sqrt{2 R^{2}\left(1+\frac{x}{R}\right)\left(1-\cos \alpha^{\prime}\right)+x^{2}+y^{2}} .
$$

With the minus sign explicitly included, the right-hand side of this equation is necessarily negative which forces $t_{r}$ to be negative, as it must be, and corresponds to picking the intersection with the lower branch in Fig. 3. This equation (implicitly) determines $\alpha^{\prime}$ as a function of $\Delta s$. Equivalently, in view of Eq. (51), the equation determines $t_{r}$ as a function of $\Delta s$. The solution of Eq. (54) for a typical choice of parameter values is shown in Fig. 7.

Two values are special: they are $s_{s}= \pm L$, at head and tail of the moving string. These values determine the extreme angles in Fig. 6 . The position defined by $\bar{\alpha}^{\prime}$ is the location at which the particle at $s_{s}=L$ has an influence at $P$ at $t=0$. Because the head particle is spatially ahead, as it arrives at $\bar{\alpha}^{\prime}$ there is still time to get a signal back to $P$ coincident with the test charge's arrival there. On the other hand a signal launched from the tail particle at $\underline{\alpha}^{\prime}$ can catch up with the test charge at $P$ by taking the path "as the crow flies." For the head particle Eq. (54) yields

$$
\frac{R \bar{\alpha}^{\prime}+s_{t}-L}{\beta}=-\sqrt{2 R^{2}\left(1+\frac{x}{R}\right)\left(1-\cos \bar{\alpha}^{\prime}\right)+x^{2}+y^{2}},
$$

and the tail equation is obtained by the replacement $-L \rightarrow L$. At least in the range $-L<s_{s}<L$, when MAPLE is instructed to find a root of this equation, it finds the correct root $\bar{\alpha}^{\prime}$. The angles found in this way are plotted in Fig. 8. 
coarse vertical scale

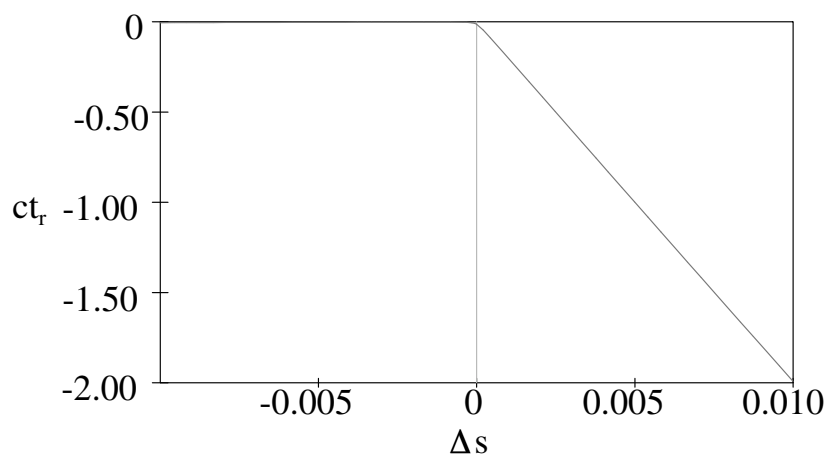

fine vertical scale

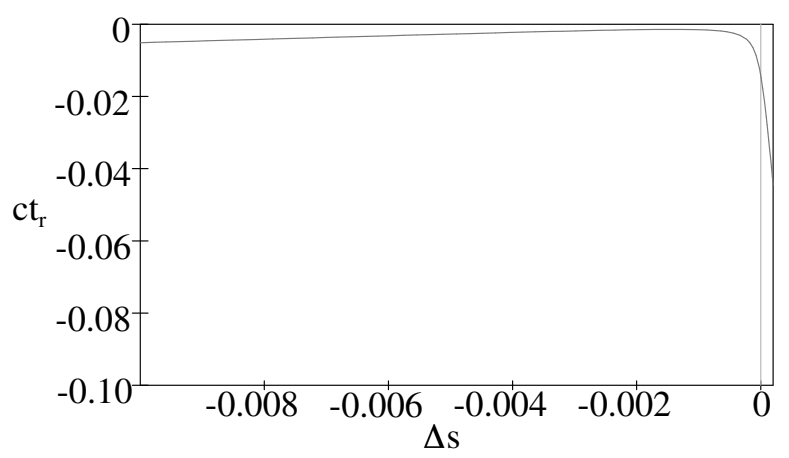

FIG. 7. Plot of $c t_{r}$ given by Eq. (54) versus $\Delta s=s_{t}-s_{s}$ with numerical values $R=80 \mathrm{~m}, x=y=1 \mathrm{~mm}$. For $2 L=0.02 \mathrm{~m}$, $s_{t}=0, \underline{\alpha}$ can be obtained from the right edge of the upper graph, $\bar{\alpha}$ from the left edge of the lower graph.

For $x=y=0$, for the end points, Eq. (54) simplifies to

$$
\begin{aligned}
& R \bar{\alpha}^{\prime}+s_{t}-L=-2 R \beta \sin \left(\bar{\alpha}^{\prime} / 2\right) \\
& R \underline{\alpha}^{\prime}+s_{t}+L=2 R \beta \sin \left(\underline{\alpha}^{\prime} / 2\right) .
\end{aligned}
$$
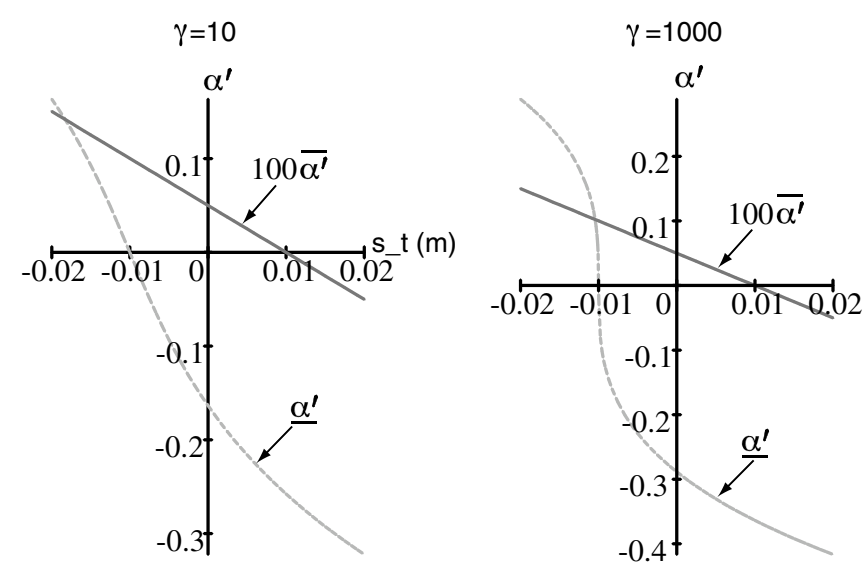

FIG. 8. The effective bunch end point angles $\underline{\alpha}^{\prime}$ and $100 \bar{\alpha}^{\prime}$, for $x=y=0$, as determined by Eqs. (56), are plotted against the test point longitudinal coordinate $s_{t} . R=10 \mathrm{~m}, L=$ $0.01 \mathrm{~m}$.
For the highly relativistic condition of interest, e.g., relativistic factor $\gamma=10^{4}$, Fig. 6 is distorted in various ways. For physical systems of interest (except in tiny intervals near the ends) one will have the following inequalities:

$$
x, y \ll s_{s} \sim s_{t} \sim \Delta s \sim L \ll R .
$$

For example, one system of interest has values $R=80 \mathrm{~m}$, $2 L=2 \mathrm{~cm}, x \sim y \sim 1 \mathrm{~mm}$. In this case $\bar{\alpha}^{\prime}$ is greatly exaggerated in the figure (and could even be negative if the head particle is only slightly longitudinally ahead of the test particle.) On the other hand it is normal for the condition $\left|\underline{\alpha} \underline{\alpha}^{\prime}\right| R \gg L$ to hold, as shown, because, with particle velocities close to the signal speed $c$, a signal from the tail particle can only catch up by "cutting across" a substantial arc.

In realistic storage ring configurations it is even possible for the shortcut just mentioned to be blocked by some obstacle, such as the inner wall of the vacuum system. This indicates that "screening effects" due to nearby conductors are likely to have significant effects on the space charge forces. Nevertheless I will neglect such things by assuming all charges are in free space. The rationale for this neglect is that the "image fields" by which such screening effects could be represented will vary but little over the extremely short transverse distances to be emphasized in the present paper. The validity of this assumption can be investigated numerically later on while applying the formulas to emittance growth. I will also neglect the longitudinal "slippage" between string charge and test charge, having identical speeds, that occurs for $x \neq 0$.

Various expansions that can be used to speed up root finding are given in the Appendix. A procedure that has been found to work uniformly is to use Eq. (A9) to find $\underline{\alpha}^{\prime}$ and the upper of Eqs. (A8) to obtain $\bar{\alpha}^{\prime}$. For production computation, direct solution of Eq. (54), with no Taylor expansion, and using sophisticated numerical rootfinding and root-polishing methods is likely to be the most robust procedure.

\section{FIELD CALCULATION}

One way of proceeding with the electromagnetic calculation would be to use the Liénard Wiechert expressions for the electric and magnetic fields of a moving point charge. Another would be to integrate over arc length the retarded time formulas for scalar and vector potentials and then to differentiate these potentials to find $\mathbf{E}$ and B-a notoriously complicated enterprise. As already stated, neither of these approaches is to be taken here. Rather $\mathbf{E}$ and $\mathbf{B}$ are to be obtained using the laws of Coulomb and Biot-Savart. In using these laws with moving charges, as well as evaluating the integrands at retarded times, it is necessary to include the extra Jefimenko terms in Eqs. (30) and (31). 
Referring to Fig. 6, with components spelled out in the order $s, x, y$,

$$
\mathbf{r}^{\prime}=\left(\begin{array}{c}
-R \sin \alpha^{\prime} \\
R\left(1-\cos \alpha^{\prime}\right)+x \\
y
\end{array}\right), \quad \hat{\mathbf{v}}^{\prime}=\left(\begin{array}{c}
\cos \alpha^{\prime} \\
-\sin \alpha^{\prime} \\
0
\end{array}\right)
$$

Note that $\mathbf{r}^{\prime}$ is the vector from retarded source point to (present) field point. The charge and current densities are to be evaluated at time $t_{r}$ calculated using Eqs. (52)-(54). The time derivative terms will be referred to as "end effect" terms, consistent with the assumption that, within the string, the charge and current densities are independent of longitudinal position and hence also of time.

For a longitudinally uniform charge distribution the charge and current densities can be expressed in terms of step function $U$;

$$
\begin{aligned}
& \lambda\left(\alpha^{\prime}\right)=\lambda_{0}\left[U\left(\alpha^{\prime}-\underline{\alpha}^{\prime}\right)-U\left(\alpha^{\prime}-\bar{\alpha}^{\prime}\right)\right] \\
& \mathbf{I}\left(\alpha^{\prime}\right)=\beta c \lambda_{0} \hat{\mathbf{v}}^{\prime}\left(\alpha^{\prime}\right)\left[U\left(\alpha^{\prime}-\underline{\alpha}^{\prime}\right)-U\left(\alpha^{\prime}-\bar{\alpha}^{\prime}\right)\right] .
\end{aligned}
$$

The only dependence of $\lambda$ on $\alpha^{\prime}$ and $t$ is through the string end locations - otherwise its value is either $\lambda_{0}$ or zero. The direction of $\mathbf{I}$ depends on $\alpha^{\prime}$, but not on time.

It is well known that there is a substantial cancellation of magnetic and electric forces between paralleltraveling, relativistic particles. It is therefore appropriate to express explicitly the force $\mathbf{F}$ acting on the test charge as it passes point $P$;

$$
\frac{\mathbf{F}(P, 0)}{e}=\mathbf{E}(P, 0)+\beta c \hat{\mathbf{s}} \times \mathbf{B}(P, 0) .
$$

Temporarily setting aside the end effect terms in Eqs. (30) and (31), and copying a manipulation due to Bassetti and Brandt [9], and using the relations $\epsilon_{0} \mu_{0}=$ $1 / c^{2}$ and $\beta^{2}=1-1 / \gamma^{2}, \hat{\mathbf{s}} \cdot \hat{\mathbf{v}}^{\prime}=\cos \alpha^{\prime}$, and $\hat{\mathbf{s}} \cdot \mathbf{r}^{\prime}=$ $-R \sin \alpha^{\prime}$, the force is given by

$$
\begin{aligned}
\frac{\mathbf{F}^{(\text {body })}}{e}= & \frac{R \lambda_{0}}{4 \pi \epsilon_{0}} \int_{\underline{\alpha}^{\prime}}^{\bar{\alpha}^{\prime}} \frac{d \alpha^{\prime}}{r^{\prime 3}}\left[\mathbf{r}^{\prime}+\beta^{2} \hat{\mathbf{s}} \times\left(\hat{\mathbf{v}}^{\prime} \times \mathbf{r}^{\prime}\right)\right] \\
= & \frac{R \lambda_{0}}{4 \pi \epsilon_{0}} \int_{\underline{\alpha}^{\prime}}^{\bar{\alpha}^{\prime}} \frac{d \alpha^{\prime}}{r^{\prime 3}}\left[\left(1-\cos \alpha^{\prime}\right) \mathbf{r}^{\prime}-R \sin \alpha^{\prime} \hat{\mathbf{v}}^{\prime}\right. \\
& \left.+\frac{1}{\gamma^{2}}\left(\cos \alpha^{\prime} \mathbf{r}^{\prime}+R \sin \alpha^{\prime} \hat{\mathbf{v}}^{\prime}\right)\right] \\
\equiv & \mathbf{F}_{0}^{\text {(body })} / e+\mathbf{F}_{1 / \gamma^{2}}^{\text {(body })} / e .
\end{aligned}
$$

In the highly relativistic $\gamma \gg 1$ domain, the force $\mathbf{F}_{1 / \gamma^{2}}^{(\text {body }}$, because it is multiplied by the small factor $1 / \gamma^{2}$, is likely to be negligible. (However, this ceases to hold in the $R \rightarrow$ $\infty$ limit.) The individual components of $\mathbf{F}_{0}^{(\text {body })}$ are

$$
\begin{aligned}
\frac{\mathbf{F}_{0}^{(\text {body })}}{e}= & \frac{R \lambda_{0}}{4 \pi \epsilon_{0}} \int_{\underline{\alpha}^{\prime}}^{\bar{\alpha}^{\prime}} \frac{d \alpha^{\prime}}{r^{\prime 3}}\left[\left(1-\cos \alpha^{\prime}\right)\right. \\
& \times\left(\begin{array}{c}
-R \sin \alpha^{\prime} \\
R\left(1-\cos \alpha^{\prime}\right)+x \\
y
\end{array}\right) \\
& \left.-R \sin \alpha^{\prime}\left(\begin{array}{c}
\cos \alpha^{\prime} \\
-\sin \alpha^{\prime} \\
0
\end{array}\right)\right] \\
= & \frac{R \lambda_{0}}{4 \pi \epsilon_{0}} \int_{\underline{\alpha}^{\prime}}^{\bar{\alpha}^{\prime}} d \alpha^{\prime}\left(\begin{array}{c}
-R \sin \alpha^{\prime} / r^{\prime 3} \\
(2 R+x)\left(1-\cos \alpha^{\prime}\right) / r^{\prime 3} \\
\left(1-\cos \alpha^{\prime}\right) y / r^{\prime 3}
\end{array}\right),
\end{aligned}
$$

and the components of $\mathbf{F}_{1 / \gamma^{2}}^{(\mathrm{body})}$ are

$$
\begin{aligned}
\frac{\mathbf{F}_{1 / \gamma^{2}}^{\text {(body })}}{e}= & \frac{R \lambda_{0}}{4 \pi \epsilon_{0} \gamma^{2}} \int_{\underline{\alpha}^{\prime}}^{\bar{\alpha}^{\prime}} \frac{d \alpha^{\prime}}{r^{\prime 3}}\left[\cos \alpha^{\prime}\left(\begin{array}{c}
-R \sin \alpha^{\prime} \\
\cos \alpha^{\prime} \\
y
\end{array}\right)\right] \\
& \left.+R \sin \alpha^{\prime}\left(\begin{array}{c}
\left.\cos \alpha^{\prime}\right)+x \\
-\sin \alpha^{\prime} \\
0
\end{array}\right)\right] \\
= & \frac{R \lambda_{0}}{4 \pi \epsilon_{0} \gamma^{2}} \\
& \times \int_{\underline{\alpha}^{\prime}}^{\bar{\alpha}^{\prime}} d \alpha^{\prime}\left(\begin{array}{c}
{\left[-R\left(1-\cos \alpha^{\prime}\right)+x \cos \alpha^{\prime}\right] / r^{\prime 3}} \\
y \cos \alpha^{\prime} / r^{\prime 3}
\end{array}\right) .
\end{aligned}
$$

The present electrodynamic calculation is closely connected (in fact patterned after) the static calculation of Bassetti and Brandt [9]. (Incidentally these authors show that $\mathbf{F}_{1 / \gamma^{2}}^{(\text {body }}$ always becomes essential as $R \rightarrow \infty$.) Equation (61) differs from the Bassetti-Brandt formula mainly because, working with a complete ring of current, their integration range is $-\pi<\alpha^{\prime}<\pi$. For the same reason their force is purely transverse. Another consequence of the newly introduced forward/back asymmetry is a nonvanishing longitudinal force. Of course, another deviation from the static calculation will result from the not-yet-included end effect forces. It has been our assumption of uniform longitudinal charge distribution that has permitted the force exhibited so far to be represented as a straightforward sum of an electrostatic, Coulomb's law force and a magnetostatic, Biot-Savart force.

In the static calculation there are charges and currents present everywhere on a circle and at all times. In the dynamic calculation these distributions vanish over most of this path, most of the time; they are nonvanishing only at times and places capable of producing fields at point $P$ at $t=0$. In either case the time-dependent charge density 
is everywhere constant, with value either 0 or $\lambda_{0}$, except at the ends of the string.

Turning to end effect terms, the step function expression in Eq. (59), with arguments fully spelled out, is

$$
U\left(\alpha^{\prime}-\frac{\beta c \underline{t}_{r}-\underline{\Delta s}}{R}\right)-U\left(\alpha^{\prime}-\frac{\beta c \bar{t}_{r}-\overline{\Delta s}}{R}\right)
$$

Time differentiation of these densities yields

$$
\begin{aligned}
& \frac{\dot{\lambda} \hat{\mathbf{r}}^{\prime}}{c r^{\prime}}=-\frac{\beta \lambda_{0}}{R r^{\prime 2}} \mathbf{r}^{\prime}\left[\delta\left(\alpha^{\prime}-\underline{\alpha}^{\prime}\right)-\delta\left(\alpha^{\prime}-\bar{\alpha}^{\prime}\right)\right], \\
& \frac{\dot{\mathbf{I}}}{c^{2} r^{\prime}}=-\frac{\beta^{2} \lambda_{0}}{R r^{\prime}} \hat{\mathbf{v}}^{\prime}\left(\alpha^{\prime}\right)\left[\delta\left(\alpha^{\prime}-\underline{\alpha}^{\prime}\right)-\delta\left(\alpha^{\prime}-\bar{\alpha}^{\prime}\right)\right] .
\end{aligned}
$$

(As mentioned before, overhead dots indicate partial derivatives with respect of time.) There is no term proportional to $\dot{\hat{\mathbf{v}}}^{\prime}$ since $\hat{\mathbf{v}}^{\prime}$ is a time-independent vector. The combination appearing in Eq. (30) is

$$
\frac{\dot{\lambda} \mathbf{r}^{\prime}}{c r^{\prime 2}}-\frac{\dot{\mathbf{I}}}{c^{2} r^{\prime}}=-\frac{\beta \lambda_{0}}{R r^{\prime 2}}\left(\mathbf{r}^{\prime}-\beta r^{\prime} \hat{\mathbf{v}}^{\prime}\right)\left[\delta\left(\alpha^{\prime}-\underline{\alpha}^{\prime}\right)-\delta\left(\alpha^{\prime}-\bar{\alpha}^{\prime}\right)\right]
$$

The end effect forces are then given by

$$
\begin{aligned}
\frac{\mathbf{F}^{(\mathrm{ends})}}{e}= & \frac{R}{4 \pi \epsilon_{0}} \int \frac{d \alpha^{\prime}}{\kappa r^{\prime 2}}\left[\frac{\dot{\lambda} \mathbf{r}^{\prime}}{c}-\frac{r^{\prime} \dot{\mathbf{I}}}{c^{2}}+\beta \hat{\mathbf{s}} \times\left(\dot{\mathbf{I}} \times \mathbf{r}^{\prime}\right)\right] \\
= & -\frac{\beta \lambda_{0}}{4 \pi \epsilon_{0}}\left\{\frac { 1 } { \kappa r ^ { \prime 2 } } \left[\mathbf{r}^{\prime}-\beta r^{\prime} \hat{\mathbf{v}}^{\prime}+\beta^{2}\left(\hat{\mathbf{s}} \cdot \mathbf{r}^{\prime}\right) \hat{\mathbf{v}}^{\prime}\right.\right. \\
& \left.\left.-\beta^{2}\left(\hat{\mathbf{s}} \cdot \hat{\mathbf{v}}^{\prime}\right) \mathbf{r}^{\prime}\right]\right]_{\bar{\alpha}^{\prime}}^{\alpha^{\prime}} \\
= & \frac{\beta \lambda_{0}}{4 \pi \epsilon_{0}}\left\{\frac { 1 } { \kappa r ^ { \prime 2 } } \left[\left(1-\cos \alpha^{\prime}\right) \mathbf{r}^{\prime}-\left(\beta r^{\prime}+R \sin \alpha^{\prime}\right) \hat{\mathbf{v}}^{\prime}\right.\right. \\
& \left.\left.+\frac{1}{\gamma^{2}}\left(\cos \alpha^{\prime} \mathbf{r}^{\prime}+R \sin \alpha^{\prime} \hat{\mathbf{v}}^{\prime}\right)\right]\right\}_{\underline{\alpha}^{\prime}}^{\bar{\alpha}^{\prime}}
\end{aligned}
$$

The denominator factor $\kappa$ is the retardation factor first introduced below Eq. (7). Here its value is

$$
\kappa=1-\beta \hat{\mathbf{v}}^{\prime} \cdot \hat{\mathbf{r}}^{\prime}=1+\beta \sin \alpha^{\prime} \frac{R+x}{r^{\prime}} .
$$

Spelling out the components, the $\gamma$-independent part is

$$
\begin{aligned}
\frac{\mathbf{F}_{0}^{(\mathrm{ends})}}{e}= & \frac{\beta \lambda_{0}}{4 \pi \epsilon_{0}}\left[\frac{1-\cos \alpha^{\prime}}{\kappa r^{\prime 2}}\left(\begin{array}{c}
-R \sin \alpha^{\prime} \\
R\left(1-\cos \alpha^{\prime}\right)+x \\
y
\end{array}\right)\right. \\
& \left.-\frac{R \sin \alpha^{\prime}+\beta r^{\prime}}{\kappa r^{\prime 2}}\left(\begin{array}{c}
\cos \alpha^{\prime} \\
-\sin \alpha^{\prime} \\
0
\end{array}\right)\right] \\
= & \frac{\beta \lambda_{0}}{4 \pi \epsilon_{0}}-R \sin \alpha^{\prime} / \kappa r^{\prime 2}-\beta \cos \alpha^{\prime} / \kappa r^{\prime} \\
& \times\left(\begin{array}{c}
(2 R+x)\left(1-\cos \alpha^{\prime}\right) / \kappa r^{\prime 2}+\beta \sin \alpha^{\prime} / \kappa r^{\prime} \\
\left(1-\cos \alpha^{\prime}\right) y / \kappa r^{\prime 2}
\end{array}\right)_{\underline{\alpha}^{\prime}}^{\bar{\alpha}^{\prime}} .
\end{aligned}
$$

The $1 / \gamma^{2}$ term in Eq. (67) will typically be negligible but, if needed, it is

$$
\frac{\mathbf{F}_{1 / \gamma^{2}}^{(\mathrm{ends})}}{e}=\frac{\beta \lambda_{0}}{4 \pi \epsilon_{0} \gamma^{2}}\left(\begin{array}{c}
0 \\
R\left(\cos \alpha^{\prime}-1\right)+x \cos \alpha^{\prime} \\
y \cos \alpha^{\prime}
\end{array}\right) \frac{1}{\kappa r^{\prime 2}} .
$$

Note the absence of longitudinal component in the $1 / \gamma^{2}$ term in both body and end contributions.

\section{THE SELF-FORCE OF A CURVING RIBBON}

For calculating the self-force we can set $x=0$, but to retain the ability to assign vertical height to the string we retain $y \neq 0$. For points exactly in the $(x=0)$ vertical cylindrical surface containing the line charge the longitudinal force can be simplified appreciably, using $r^{\prime}=$ $\sqrt{2 R^{2}}\left(1-\cos \alpha^{\prime}\right)+y^{2}$, to yield, for the body contribution,

$$
\begin{aligned}
\frac{F_{s}^{\text {body })}}{e}= & \frac{R \lambda_{0}}{4 \pi \epsilon_{0}} \int_{\underline{\alpha}^{\prime}}^{-\bar{\alpha}^{\prime}} d \alpha^{\prime} \frac{-R \sin \alpha^{\prime}}{\left[2 R^{2}\left(1-\cos \alpha^{\prime}\right)+y^{2}\right]^{3 / 2}} \\
= & \frac{\lambda_{0}}{4 \pi \epsilon_{0}}\left[\frac{1}{\left[2 R^{2}\left(1-\cos \alpha^{\prime}\right)+y^{2}\right]^{1 / 2}}\right]_{\underline{\alpha}^{\prime}}^{\bar{\alpha}^{\prime}} \\
= & \frac{\lambda_{0}}{4 \pi \epsilon_{0}}\left[-\frac{\beta}{R \bar{\alpha}^{\prime}+\overline{\Delta s}}+\frac{\beta}{R \underline{\alpha}^{\prime}+\underline{\Delta s}}\right] \stackrel{\text { also }}{=} \frac{\lambda_{0}}{4 \pi \epsilon_{0}} \\
& \times\left(\frac{1}{\bar{r}^{\prime}}-\frac{1}{\underline{r}^{\prime}}\right) .
\end{aligned}
$$

In the second-to-last expression Eq. (54) has been used and the signs have been chosen as in Eq. (56). The final expression, which uses $\overline{\Delta s}=s_{t}-L$ and $\underline{\Delta s}=s_{t}+L$, can be seen to agree with Eq. (20) in the $y=0$, nocurvature limit. Using $r^{\prime}=y / \sin \theta^{\prime}$, it also agrees with the $z$ component of Eq. (33). The end contributions to longitudinal force are 


$$
\begin{aligned}
\frac{F_{s}^{(\mathrm{ends})}}{e}= & -\frac{\lambda_{0}}{4 \pi \epsilon_{0}}\left\{\left[\frac{\beta^{2} \cos \alpha^{\prime} / \kappa}{r^{\prime}}\right]_{\underline{\alpha}^{\prime}}^{\bar{\alpha}^{\prime}}+\frac{R \beta \sin \bar{\alpha}^{\prime} / \bar{\kappa}}{\bar{r}^{\prime 2}}\right. \\
& \left.-\frac{R \beta \sin \underline{\alpha}^{\prime} / \underline{\kappa}}{\underline{r}^{\prime 2}}\right\} .
\end{aligned}
$$

From Eq. (68), in this case

$$
\bar{\kappa}=1+\frac{\beta R \sin \bar{\alpha}^{\prime}}{\bar{r}^{\prime}}, \quad \underline{\kappa}=1+\frac{\beta R \sin \underline{\alpha}^{\prime}}{\underline{r}^{\prime}} .
$$

In the $R \rightarrow \infty, y \neq 0$ case, $R \sin \alpha^{\prime} \rightarrow-r^{\prime} \cos \theta^{\prime}$, and Eq. (72) agrees with the $z$ component of Eq. (34).

Summing the body and end terms yields

$$
\begin{aligned}
\frac{F_{s}^{(R)}\left(s_{t}, 0, y\right)}{e}= & \frac{\lambda_{0}}{4 \pi \epsilon_{0}}\left(\frac{1-\beta^{2} \cos \bar{\alpha}^{\prime} / \bar{\kappa}}{\bar{r}^{\prime}}-\frac{R \beta \sin \bar{\alpha}^{\prime} / \bar{\kappa}}{\bar{r}^{\prime 2}}\right. \\
& \left.-\frac{1-\beta^{2} \cos \underline{\alpha}^{\prime} / \underline{\kappa}}{\underline{r}^{\prime}}+\frac{R \beta \sin \underline{\alpha}^{\prime} / \underline{\kappa}}{\underline{r}^{\prime 2}}\right) \\
= & \frac{\lambda_{0}}{4 \pi \epsilon_{0}}\left(\frac{1-\beta^{2} \cos \bar{\alpha}^{\prime}}{\bar{r}^{\prime}+\beta R \sin \bar{\alpha}^{\prime}}-\frac{1-\beta^{2} \cos \underline{\alpha}^{\prime}}{\underline{r}^{\prime}+\beta R \sin \underline{\alpha}^{\prime}}\right) .
\end{aligned}
$$

The superscript $(R)$ has been attached for a reason to be explained shortly. In the $R \rightarrow \infty$ limit, with $y=0$, $R \sin \alpha^{\prime} \rightarrow r^{\prime}$, and this formula reduces to Eq. (10). These formulas continue to be valid for points on the circular arc but located outside the range $-L<s_{t}<L$. Also, for $y \neq 0$, the $y^{2}$ term in the denominator will suppress the divergences at the end points when calculating the total force acting on the string. In applying these formulas the upper and lower limits have to be determined numerically in general. But for points with $x=$ $y=0$, exactly on the (curved) line charge, the limits can be evaluated as described earlier. The first term denominator of Eq. (74), especially for $s_{t} \gtrsim L$, requires great numerical precision to be evaluated accurately.

It is possible to express the force more nearly in terms of present-time coordinates using the following manipulations which replicate the treatment following Eq. (35). Referring to Fig. 9, the tail retardation condition is

$$
\begin{aligned}
& L+s_{t}+R \underline{\alpha}^{\prime}=-\beta \underline{r}^{\prime} \quad \text { or } \\
& \quad L+s_{t}+R\left(\underline{\alpha}^{\prime}-\sin \underline{\alpha}^{\prime}\right)=-\left(\beta \underline{r}^{\prime}+R \sin \underline{\alpha}^{\prime}\right) .
\end{aligned}
$$

As in Eq. (38), the tail denominator can be written as

$$
\underline{r}^{\prime}+\beta R \sin \underline{\alpha}^{\prime}=\sqrt{\underline{r}^{\prime 2}+2 \beta \underline{r}^{\prime} R \sin \underline{\alpha}^{\prime}+\beta^{2} R^{2} \sin ^{2} \underline{\alpha}^{\prime}+R^{2} \sin ^{2} \underline{\alpha}^{\prime}-R^{2} \sin ^{2} \underline{\alpha}^{\prime}+\beta^{2} \underline{r}^{\prime 2}-\beta^{2} \underline{r}^{\prime 2}} .
$$

Under the square root, the sum of the second, fourth, and sixth terms can be recognized, using Eq. (75), as $\left[L+s_{t}+\right.$ $\left.R\left(\underline{\alpha}^{\prime}-\sin \underline{\alpha}^{\prime}\right)\right]^{2}$ and the remaining terms sum to

$$
\left(\underline{r}^{\prime 2}-R^{2} \sin ^{2} \alpha^{\prime}\right)\left(1-\beta^{2}\right)=\left\{y^{2}+\left[x+R\left(1-\cos \underline{\alpha}^{\prime}\right)\right]^{2}\right\} / \gamma^{2} .
$$

As a result,

$$
\underline{r}^{\prime}+\beta R \sin \underline{\alpha}^{\prime}=\sqrt{\left[L+s_{t}+R\left(\underline{\alpha}^{\prime}-\sin \underline{\alpha}^{\prime}\right)\right]^{2}+\left\{y^{2}+\left[x+R\left(1-\cos \underline{\alpha}^{\prime}\right)\right]^{2}\right\} / \gamma^{2}} .
$$

For short strings one can use the approximation

$$
\underline{\alpha}^{\prime}-\sin \underline{\alpha}^{\prime} \approx \alpha^{\prime 3} / 6
$$

For the head of the string the retardation condition is

$$
-L+s_{t}+R \bar{\alpha}^{\prime}=-\beta \bar{r}^{\prime} \quad \text { or } \quad-L+s_{t}+R\left(\bar{\alpha}^{\prime}-\sin \bar{\alpha}^{\prime}\right)=-\left(\beta \bar{r}^{\prime}+R \sin \bar{\alpha}^{\prime}\right),
$$

and the denominator factor can also be rearranged. Substituting these relations, the longitudinal force component is

$$
\begin{aligned}
\frac{F_{s}\left(s_{t}, 0, y\right)}{e}= & \frac{\lambda_{0}}{4 \pi \epsilon_{0}}\left(\frac{1-\beta^{2} \cos \bar{\alpha}^{\prime}}{\sqrt{\left[-L+s_{t}+R\left(\bar{\alpha}^{\prime}-\sin \bar{\alpha}^{\prime}\right)\right]^{2}+\left[\bar{y}^{2}+R^{2}\left(1-\cos \bar{\alpha}^{\prime}\right)^{2}\right] / \gamma^{2}}}\right. \\
& \left.-\frac{1-\beta^{2} \cos \underline{\alpha}^{\prime}}{\sqrt{\left[L+s_{t}+R\left(\underline{\alpha}^{\prime}-\sin \underline{\alpha}^{\prime}\right)\right]^{2}+\left[y^{2}+R^{2}\left(1-\cos \underline{\alpha}^{\prime}\right)^{2}\right] / \gamma^{2}}}\right) .
\end{aligned}
$$

\section{RENORMALIZATION OF THE LONGITUDINAL FORCE}

It was established earlier that there is no net selfforce for a straight string in a field-free region. This largely obviates the need for any renormalization. But for a curved string, even if it is subject to zero acceleration, since the ends are not quite parallel, a "small" logarithmically divergent self-force seems likely to survive. Furthermore, the presence of divergent terms at string ends, even if they sum to zero in the total force, prevent the internal forces from being calculated reliably. For calculating effects due entirely to acceleration, forces present even with acceleration absent need to be subtracted or otherwise accounted for. 


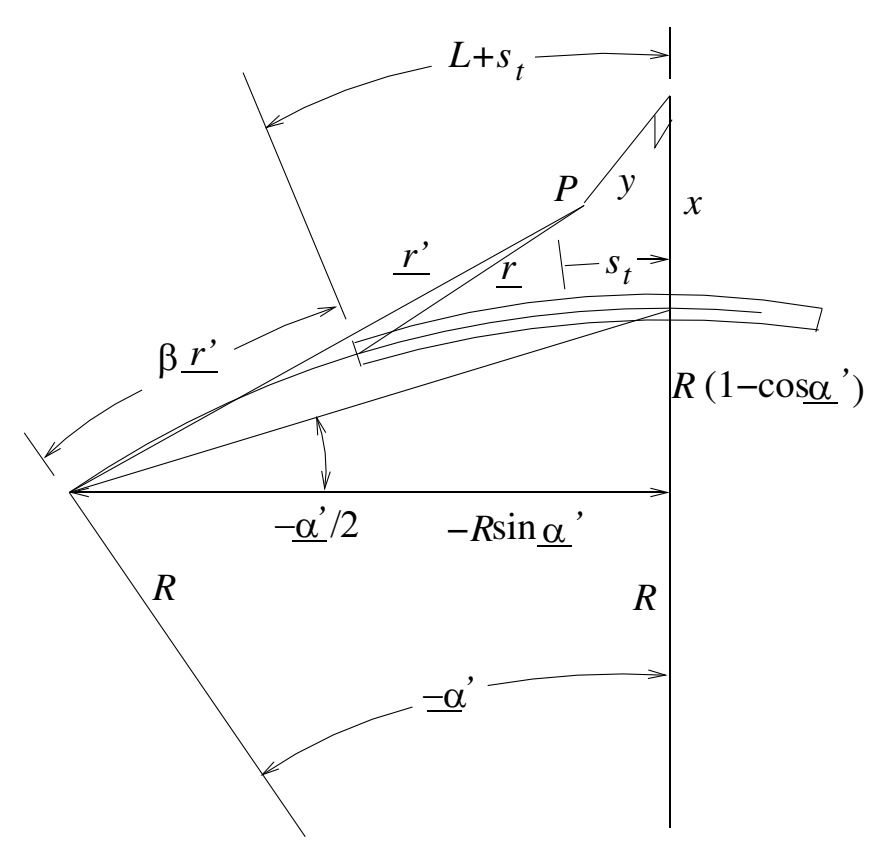

FIG. 9. Geometry for interpreting short bunch approximations.

The straight string field calculated in Eq. (35) should be derivable from the formulas just derived as a special case. To do this let us change the variable of integration from $\alpha^{\prime}$ to $s^{\prime}=\alpha^{\prime} R$ using $\cos \alpha^{\prime} \rightarrow \pm 1$ and $R \sin \alpha^{\prime} \rightarrow$ $-r^{\prime} \cos \theta^{\prime}$, where $\theta^{\prime}$ is an angle shown in Fig. 5. As a result,

$$
\begin{aligned}
\frac{F_{s}^{(\infty)}\left(s_{t}, 0, y\right)}{e}= & \lim \frac{\lambda_{0}}{4 \pi \epsilon_{0}}\left(\frac{1-\beta^{2} \cos \bar{\alpha}^{\prime}}{\bar{r}^{\prime}+\beta R \sin \bar{\alpha}^{\prime}}\right. \\
& \left.-\frac{1-\beta^{2} \cos \underline{\alpha}^{\prime}}{\underline{r}^{\prime}+\beta R \sin \underline{\alpha}^{\prime}}\right) \\
= & \frac{\lambda_{0}}{4 \pi \epsilon_{0}}\left(\frac{1 / \gamma^{2}}{\bar{r}^{\prime}\left(1-\beta \cos \bar{\theta}^{\prime}\right)}\right. \\
& \left.-\frac{1 / \gamma^{2}}{\underline{r}^{\prime}\left(1-\beta \cos \underline{\theta}^{\prime}\right)}\right) .
\end{aligned}
$$

This formula agrees with Eq. (35). For $y=0$ the end points are given by Eq. (14).

We are now in a position to perform the renormalization process mentioned in the Introduction. Symbolizing the curvature-present force by $\mathbf{F}^{(R)}$, the curvature-absent force by $\mathbf{F}^{(\infty)}$, and their difference by $\mathbf{G}$, one has

$$
\mathbf{F}^{(R)}=\mathbf{F}^{(\infty)}+\mathbf{G}, \quad \text { where } \mathbf{G}=\mathbf{F}^{(R)}-\mathbf{F}^{(\infty)} .
$$

The definitions of these terms is made more explicit by referring to Fig. 10. Force $\mathbf{F}^{(R)}(x, y, s)$ at position $P$ is to be calculated based on the curved charge distribution running from $\underline{s}_{R}$ to $\bar{s}_{R}$ and force $\mathbf{F}(\infty)(x, y, s)$ is to be calculated based on the straight charge distribution running from $\underline{s}_{\infty}$ to $\bar{s}_{\infty}$. For transverse positions $x, y$ very close to either end of the charge distribution $\mathbf{F}^{(R)}$ and $\mathbf{F}^{(\infty)}$ will exhibit the same logarithmic divergence. As a result the divergence will be absent from $\mathbf{G}$.

The renormalized force $\mathbf{G}$ can be used to calculate the emittance growth due purely to orbit curvature. But there may also be emittance growth caused by space charge forces even in field-free regions. That growth will have to be estimated using $\mathbf{F}$, in which case the divergence has to be handled differently. The intention of treating point particles as strings is to obviate the need for renormalization. By starting all particles in a simulation with nonzero interparticle separation the divergence can only occur after some bunch evolution has occurred, and then the probability of exact spatial coincidence later on is small, especially for high precision computation. The occasional close encounter will be insignificant since the singularity is only logarithmic. Of course these comments have to be made quantitative by numerical investigation.

Sample evaluations of the longitudinal force are shown in Fig. 11. For purposes of comparison values due to Saldin et al. [10] are also plotted. The lower $\gamma$ value is close to, and the higher much greater than, the energy above which Eq. (88) becomes a good approximation. It can be seen that the agreement with Saldin is excellent

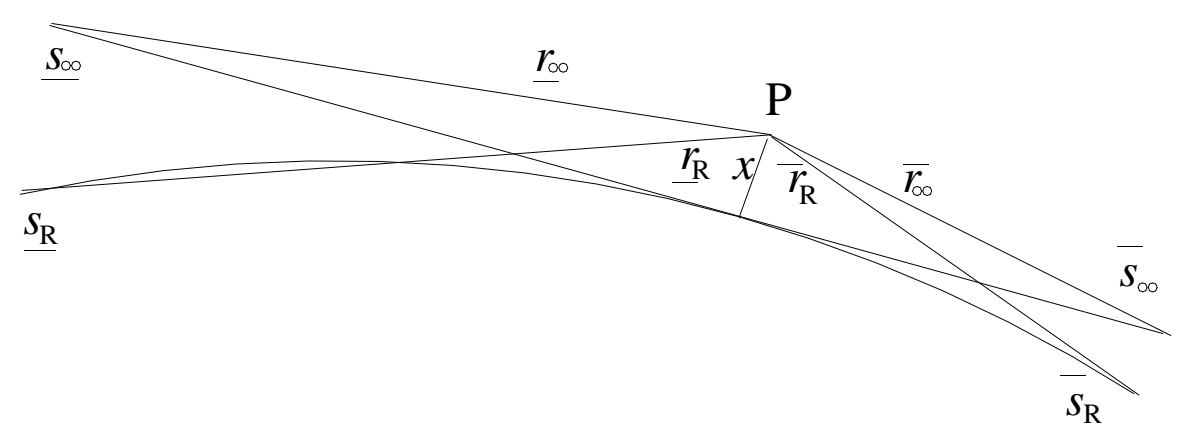

FIG. 10. Superposition of straight line and curved charge distribution for the purpose of renormalizing the self-force due to the curvature of the path. At each point $P$ the renormalized force $\mathbf{G}$ is obtained by subtracting the tangentially matched straight line force $\mathbf{F}^{(\infty)}$ from the curved line force $\mathbf{F}^{(R)}$. 


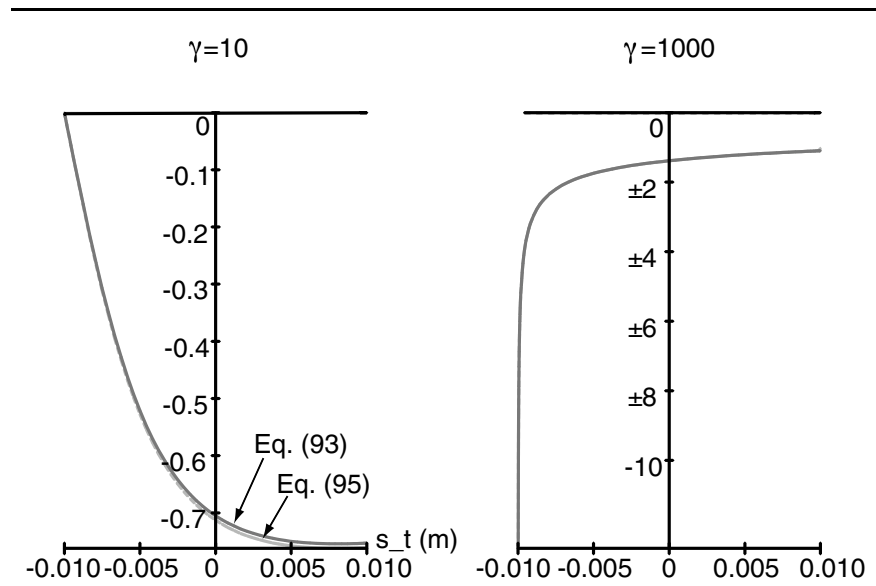

FIG. 11. Plot of $G_{s}\left(s_{t}, 0,0\right)$, for $R=10 \mathrm{~m}, L=0.01 \mathrm{~m}$, for two $\gamma$ values. In both cases the result from this paper, Eq. (83), is plotted as a solid line. The factor $\left(e \lambda_{0}\right) /\left(4 \pi \epsilon_{0}\right)$ is suppressed in this and the next plot. For $\gamma=10$ the exact Saldin formula (87) is plotted as a broken line. For $\gamma=1000$ the high-energy approximate formula (88), $G_{s}^{\left(\gamma \gg 1 / u_{s}\right)}$, is plotted as a broken line. Especially for the $\gamma=1000$ graph, the curves superimpose well enough to be scarcely distinguishable.

and that the $G_{s}^{\left(\gamma \gg 1 / u_{s}\right)}$ approximation is excellent at large $\gamma$. But this approximation, independent of $\gamma$ as it is, greatly overestimates the self-force (and hence the CSR) at $\gamma=10$. For the same two $\gamma$ values the unrenormalized longitudinal force $F_{s}$ is exhibited for both curving and straight strings in Fig. 12. Here the longitudinal range is extended both before and after the actual string. The vertical axis has been artificially distorted (cube root) in order to expand the dynamic range while leaving the internal force visible.

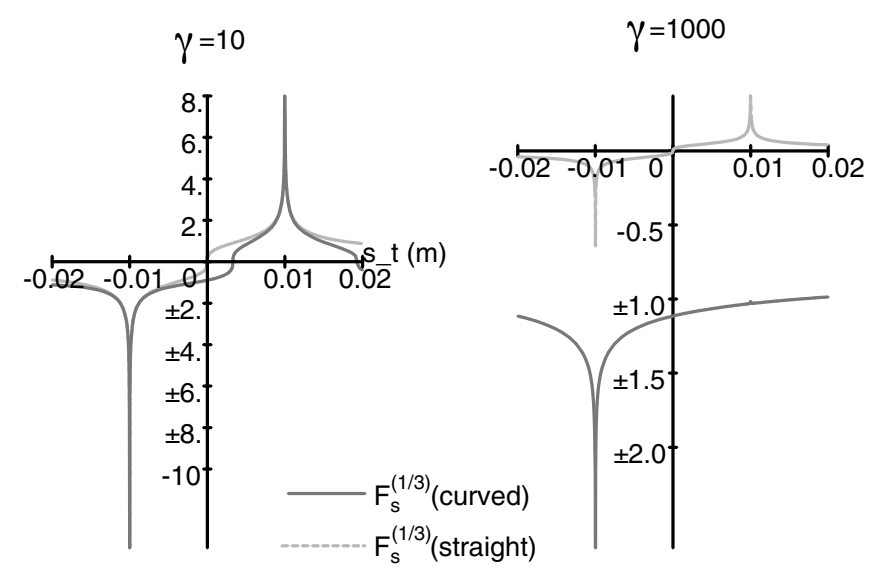

FIG. 12. The curved string and straight string longitudinal forces (after taking their cube roots) are shown over a range extended in front of and behind the string. (There is nothing fundamental about cube root; this function has been chosen only to expand the scale at low amplitude relative to that at large amplitude while otherwise preserving the general shape.) As $\gamma$ increases, the straight string force becomes negligible compared to the curving string case. $R=10 \mathrm{~m}, L=0.01 \mathrm{~m}$.

\section{COHERENT SYNCHROTRON RADIATION}

The power radiated by $N$ electrons in the form of synchrotron radiation has been calculated by Schwinger [16], both in the form of coherent and incoherent radiation:

$$
\begin{aligned}
P_{\mathrm{coh}}^{(N)} & =\beta N^{2} \frac{c e^{2}}{4 \pi \epsilon_{0} R^{2}}\left(\frac{\sqrt{3} R}{2 L}\right)^{4 / 3}, \\
P_{\text {incoh }}^{(N)} & =\beta N \frac{2}{3} \frac{c e^{2}}{4 \pi \epsilon_{0} R^{2}} \gamma^{4} .
\end{aligned}
$$

These contributions are equal for

$$
\gamma_{\text {crit }}=\left(\frac{3 N}{2}\right)^{1 / 4}\left(\frac{\sqrt{3} R}{2 L}\right)^{1 / 3}
$$

The coherent power radiated should be equal to the rate at which the bunch does work on itself, which is given by

$$
P=\beta c \int_{-L}^{L} \frac{G_{s}\left(s_{t}, 0,0\right)}{e} \lambda_{0} d s_{t},
$$

where $\lambda_{0}=N e /(2 L)$.

The on-axis renormalized longitudinal field $G_{s}$ can be compared with results of Saldin et al. [10]. For this comparison, our $s_{t}=s_{\text {Saldin }}+L$, and their symbol $u_{s}$, in our notation, is

$$
u_{s} \equiv \underline{\alpha}^{\prime} .
$$

They give the formula

$$
\frac{G_{s}\left(s_{t}, 0,0\right)}{e}=-\frac{\lambda_{0}}{4 \pi \epsilon_{0}} \frac{4 \gamma}{R} \frac{\left(\gamma \underline{\alpha}^{\prime}\right)\left(8+\gamma^{2} \underline{\alpha}^{\prime 2}\right)}{\left(4+\gamma^{2} \underline{\alpha}^{\prime 2}\right)\left(12+\gamma^{2} \underline{\alpha}^{\prime 2}\right)} .
$$

This reduces to an approximate form, valid for $\gamma \gg 1 / u_{s}$ which, expressed in our notation, is

$$
\frac{G_{s}^{\left(\gamma \gg 1 / u_{s}\right)}}{e}=\frac{\lambda_{0}}{4 \pi \epsilon_{0}} \frac{2}{3^{1 / 3}} \frac{1}{R^{2 / 3}} \frac{1}{\left(L+s_{t}\right)^{1 / 3}} .
$$

This approximation, which, after integration from $-L$ to $L$, gives perfect agreement with Eq. (84) for $P_{\text {coh }}^{(N)}$, is given by Saldin et al. [10] only in the range $-L<s_{t}<L$.

Some numerical comparisons of the two methods of determining coherent power are given in Table I. High-

TABLE I. Coherent power, as calculated by self-force $P$ and as by Schwinger from far fields, $P_{\mathrm{coh}}^{(N)}$.

\begin{tabular}{lccccc}
\hline \hline$L(\mathrm{~m})$ & $R(\mathrm{~m})$ & $\gamma$ & $P(\mathrm{~W})$ & $P_{\text {coh }}^{(N)}(\mathrm{W})$ & $\gamma_{\text {crit }}$ \\
\hline 0.01 & 10 & 10 & 207 & 568 & 3336 \\
& & 100 & 555 & 571 & 3336 \\
& & 1000 & 563 & 571 & 3336 \\
& & 10000 & 563 & 571 & 3336 \\
0.001 & 10 & 1000 & 12179 & 12298 & 7187 \\
0.01 & 100 & 1000 & 121.8 & 123.0 & 7187 \\
\hline \hline
\end{tabular}


energy approximation (88) becomes an excellent approximation only for surprisingly large values of $\gamma$, such as $\gamma=100$. As explained by Saldin et al. [10], roughly speaking, the approximation is valid only for $\gamma \gg$ $(R / L)^{1 / 3}$. (The roughly $1 \%$ disagreement of $P$ and $P_{\mathrm{coh}}^{(N)}$ for ultralarge value such as $\gamma=10000$ is presumably ascribable to the only-rudimentary evaluation of the integral by parallelogram rule - a more accurate integration prescription was unable to handle the singular behavior of the integrand at the end points.)

In summary, as stated in the Introduction, agreement with on-axis results of Saldin et al. is excellent.

\section{EFFECTS OF ENTERING AND LEAVING MAGNETS}

Any practical accelerator consists of alternating bend and drift regions. As a result the space charge force due to a moving string needs special treatment at entrances and exits of bending regions. In the procedure being described substantial calculational complication. For calculating the force on a particular particle the first thing to be calculated is the ends of the effective (i.e., retarded) charge distribution. If the field point is inside a magnet one or the other of the effective ends may be outside, or vice versa. We have seen that the effective head is very close to the field point, so it seems to be a good approximation to declare this always to be the case. This reduces the problem to locating the effective tail. If this location is in the same magnetic element as the field point, be it drift or bend, the force is given by one of the formulas derived in this paper.

Suppose the effective charge distribution crosses a field boundary. (In principle it could cross more than one but, for simplicity, let us ignore that possibility.) By calculation one can therefore locate the point along the effective charge distribution that coincides with the boundary. It is natural then to break the string at that point into a head segment and a tail segment. Within the head segment, the analytic retarded time formula being used is valid, so the this presents little conceptual difficulty, but it does lead to

force due to the front segment can be calculated directly, using curved or straight string as appropriate. The only substantial new calculation is to locate the tail of the tail segment. Though straightforward this will require formulas not given in this paper. Once the ends of the tail segment have been located, the tail segment's contribution to the force can be obtained using formulas from this paper. This contribution is likely to be quite different from what would be given by treating the entire effective charge distribution as if in the same field as the field point.

An effect that is likely to be as important as that just described is the shielding effect of nearby vacuum chamber walls. This effect has been neglected so far. Like the two-region problem of the previous paragraph, this possibility affects only the tail contribution of the effective charge distribution. The prescription I suggest starts by finding whether a straight line from tail point to field point intersects the chamber wall. If it does not the wall can be ignored. But, if there is an intersection, a tangency condition can be applied to identify the point along the string where intersection first occurs. Segmenting the string at that point, the tail segment can simply be dropped and the total force given by the standard formulas applied to the front segment. As discussed earlier, this procedure neglects diffractive and image effects.

\section{EVALUATION OF INTEGRALS}

The end effect forces have been given in closed form and the longitudinal body force integral evaluated for $x=$ 0 . For $x \neq 0$ the integrals can also be evaluated in closed form, but they are more complicated. Depending, as they do, on $r^{\prime}$ as given by Eq. (53), the integrals appearing in Eqs. (62) and (63) depend on factors of the form $\sqrt{A+B \cos \alpha^{\prime}}$ where, satisfying $A>0$ and $A+B>0$,

$$
A \equiv 2 R(R+x)+x^{2}+y^{2}, \quad B \equiv-2 R(R+x) .
$$

(Using, for example, MAPLE) the required integrals can be expressed in terms of incomplete elliptic integrals [17]. (The MAPLE argument $k$ is related to the Abramowitz and Stegun argument $m$ by $m=k^{2}$.)

$$
\begin{gathered}
\mathrm{E}(z, k)=\int_{0}^{z} \sqrt{1-k^{2} t^{2}} / \sqrt{1-t^{2}} d t, \quad \mathrm{~F}(z, k)=\int_{0}^{z} 1 / \sqrt{\left(1-k^{2} t^{2}\right)\left(1-t^{2}\right)} d t, \\
\operatorname{Pi}(z, \nu, k)=\int_{0}^{z} 1 / \sqrt{\left(1-\nu t^{2}\right)\left(1-t^{2}\right)\left(1-k^{2} t^{2}\right)} d t, \\
I_{s}=\int \frac{\sin \alpha^{\prime}}{\left(A+B \cos \alpha^{\prime}\right)^{3 / 2}} d \alpha^{\prime}=\frac{2}{B \sqrt{A+B \cos \alpha^{\prime}}}, I_{0}=\int \frac{\cos \alpha^{\prime}}{\left(A+B \cos \alpha^{\prime}\right)^{3 / 2}} d \alpha^{\prime}, \\
I_{x, y}=\int \frac{1-\cos \alpha^{\prime}}{\left(A+B \cos \alpha^{\prime}\right)^{3 / 2}} d \alpha^{\prime} \\
=2 \frac{-B \sin \alpha^{\prime} \sin \left(\alpha^{\prime} / 2\right)+\sqrt{1-\cos ^{2}\left(\alpha^{\prime} / 2\right)} \sqrt{A-B} \sqrt{A-B+2 B \cos ^{2}\left(\alpha^{\prime} / 2\right)}\left[\mathrm{F}\left(\cos \left(\alpha^{\prime} / 2\right), \sqrt{(-2 B) /(A-B)}\right)-\mathrm{E}(\cdots)\right]}{\sin \left(\alpha^{\prime} / 2\right) \sqrt{A-B+2 B \cos ^{2}\left(\alpha^{\prime} / 2\right)} B(A-B)},
\end{gathered}
$$


where both elliptic functions have the same arguments. Though not used in this paper, to represent a vertical ribbon of charge, it is even possible to integrate first over $y$;

$$
\begin{aligned}
& I I_{s}=\int d \alpha^{\prime} \int d y \frac{\sin \alpha^{\prime}}{\left(A+B \cos \alpha^{\prime}\right)^{3 / 2}}=\frac{1}{2 R(R+x)} \ln \frac{\sqrt{2 R(R+x)+x^{2}+y^{2}-2 R(R+x) \cos \alpha^{\prime}}-y}{\sqrt{2 R(R+x)+x^{2}+y^{2}-2 R(R+x) \cos \alpha^{\prime}}+y}, \\
& I I_{x}=\int d \alpha^{\prime} \int d y \frac{1-\cos \alpha^{\prime}}{\left(A+B \cos \alpha^{\prime}\right)^{3 / 2}}=-y \frac{x^{2}\left[\mathrm{~F}\left(\cos \left(\alpha^{\prime} / 2\right), D\right)-\operatorname{Pi}\left(\cos \left(\alpha^{\prime} / 2\right), C, D\right)\right]+4 R(R+x) \mathrm{F}\left(\cos \left(\alpha^{\prime} / 2\right), D\right)}{R(R+x)(x+2 R)^{2} \sqrt{4 R(R+x)+x^{2}+y^{2}}},
\end{aligned}
$$

where

$$
C=\frac{4 R(R+x)}{(x+2 R)^{2}}, \quad D=2 \sqrt{\frac{R(R+x)}{4 R(R+x)+x^{2}+y^{2}}} .
$$

Several points can be made about these formulas.

(i) $I_{s}$ and $I_{x, y}$ give, respectively, the $s$ and the $x$ or $y$ components of the force produced by a string of charge acting on a point charge.

(ii) $I I_{s}$ and $I I_{x}$ give the components of the force of a vertical ribbon on a point charge. No formula for $I I_{y}$ is given; the vertical force is presumably small.

(iii) Since $I I_{x}$ is so complicated it may have typographical errors or may acquire them when transcribed; it is exhibited primarily to indicate its degree of complexity. If $I I_{x}$ is needed, it should be regenerated automatically using a mathematical programming language. In this paper it has been used only as a selfconsistency check.

(iv) Integration over an extended bunch can be formulated as a one-dimensional array of ribbons, using the $I I$ integrals, or as a two dimensional array of strings using the $I$ integrals. Each has advantages and disadvantages.

(v) Though less singular than the force between point charges, the force due to a string is singular for charges exactly on the string, $x=y=0$. To circumvent this problem during summation over the transverse bunch distribution the $x, y$ field point grid can be "staggered" relative to the $x, y$ source point grid. Since the singularity is only logarithmic this is probably satisfactory.

(vi) Each integral that is performed analytically reduces the singularity of the force for small $x$ and $y$, so the II functions are not singular. Unfortunately the end points of the $\alpha^{\prime}$ integration $\left(\underline{\alpha}^{\prime}\right.$ and $\left.\bar{\alpha}^{\prime}\right)$ are functions of $y$. It may be that this dependency is unimportant, but it seriously complicates the evaluation of the force between ribbon and point charge.

There is a serious hazard to be avoided in applying these formulas. It is that, as functions of $\alpha^{\prime}$, and depending on their ranges of definition, the elliptic-integral expressions may be discontinuous at $\alpha^{\prime}=0$. It is therefore appropriate, in every case for which $\alpha^{\prime}=0$ lies in the range of integration, to break the integration into two ranges, one terminating just before the origin, the other beginning just after the origin.

\section{FORCE ON POINT CHARGE DUE TO DISTRIBUTION OF CHARGED STRINGS}

The (vector) force on a test charge due to a string of charge has been derived in previous sections and a consistent renormalization procedure described for the longitudinal component. Since this procedure suppresses the zero-curvature space charge force, it is worth investigating the extent to which renormalization is really required. That is the purpose for Fig. 12, which shows, for a particular parameter set, that the renormalization is quite insignificant. This section proposes a numerical procedure intended to give a good approximation even with renormalization neglected. For simplicity the $\mathbf{F}_{1 / \gamma^{2}}$ terms will not be exhibited, even though they may be required for even quite relativistic bunches.

The initial conditions of a transversely extended bunch of charge will be represented by a two dimensional transverse distribution of parallel charged strings. (The restriction to a uniform transverse grid of sources is not at all fundamental. It is made only to simplify this paper.)

Forces are calculated at the grid points shown in Fig. 13 with $x_{t}-x_{s}$ plotted horizontally and $y_{t}-y_{s}$ vertically. The repetition periods are $2 g$ horizontally and $2 h$ vertically, and the field grid points are staggered by a half-period in both transverse directions so they cannot coincide with the source point. The longitudinal grid spacing (not shown) is $\ell$.

Test charge grid locations relative to source charge are to be indexed as

$$
\begin{gathered}
x^{I}=I g, \quad y^{J}=J h, \\
\underline{\Delta s}^{K}=K \ell+L, \quad \overline{\Delta s}^{K}=K \ell-L,
\end{gathered}
$$

with $I$ and $J$ both odd. By symmetry, values need only be calculated in one quadrant, but interpolation will, in general, combine values from more than one quadrant.

The string tail equation is

$$
\begin{aligned}
& R \underline{\alpha}^{\prime I J K}+\underline{\Delta s}^{K} \\
& \quad=-\beta \sqrt{R^{2}+\left(R+x^{I}\right)^{2}+y^{J 2}-2 R\left(R+x^{I}\right) \cos \underline{\alpha}^{I J K}},
\end{aligned}
$$

and the string head equation is 
radially outward

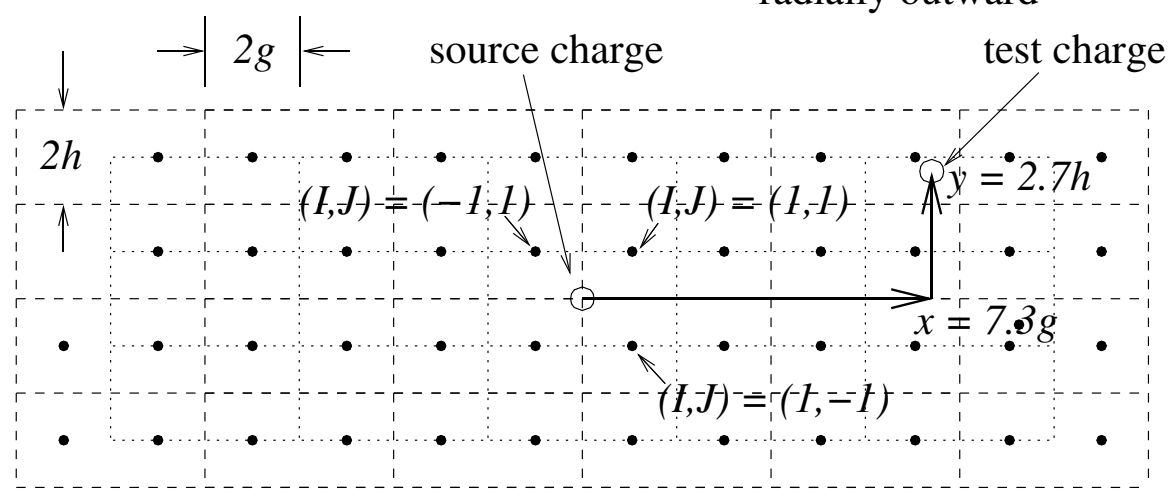

FIG. 13. Transverse positions of field point relative to source point, $x_{t}-x_{s}$ plotted horizontally and $y_{t}-y_{s}$ vertically. Fields are calculated at the dot-dot grid intersections, which are half-period staggered relative to the source point.

$$
\begin{aligned}
& R \bar{\alpha}^{I I J K}+\overline{\Delta s} K \\
& \quad=-\beta \sqrt{R^{2}+\left(R+x^{I}\right)^{2}+y^{J 2}-2 R\left(R+x^{I}\right) \cos \bar{\alpha}^{I I J K}} .
\end{aligned}
$$

As mentioned earlier, because the parameters in these equations are so extreme, the equations are likely to require delicate solution methods.

Substituting into Eq. (62), dropping the factor $\lambda_{0} /\left(4 \pi \epsilon_{0}\right)$, the fully relativistic components of force (not yet including end contributions) are

$$
\begin{gathered}
\left(F_{0}^{I J K}\right)_{s}^{(\mathrm{body})}=-R^{2}\left[I_{s}\left(A^{I J}, B^{I}\right)\right]_{\underline{\alpha}^{\prime I J K}}^{\bar{\alpha}^{\prime I J K},} \\
\left(F_{0}^{I J K}\right)_{x}^{(\mathrm{body})}=R\left(2 R+x^{I J K}\right)\left[I_{x, y}\left(A^{I J}, B^{I}\right)\right]_{\underline{\alpha}^{\prime I J K}}^{0-} \\
+R\left(2 R+x^{I J K}\right)\left[I_{x, y}\left(A^{I J}, B^{I}\right)\right]_{0+}^{\bar{\alpha}^{\prime I J K}}, \\
\left(F_{0}^{I J K}\right)_{y}^{(\mathrm{body})}=R y^{J}\left[I_{x, y}\left(A^{I J}, B^{I}\right)\right]_{\underline{\alpha}^{\prime I J K}}^{\bar{\alpha}^{\prime I J K}}
\end{gathered}
$$

Recall, however, as explained above, that ranges including the origin have to be treated as two separate intervals, at least in the transverse cases. This is indicated (schematically) only in the second equation which, technically, is only correct if the origin lies in the range. To be safe none of the expressions should be used for ranges including the origin, and the upper limits should be required to be more positive than the lower limits.

From Eqs. (67) and (69) the end effect force components are

$$
\begin{aligned}
& \left(F_{0}^{I J K}\right)_{s}^{(\text {ends) }}=\beta\left[\frac{R \sin \alpha^{\prime}}{\kappa r^{I J K 2}}+\frac{\beta \cos \alpha^{\prime}}{\kappa r^{I J K}}\right]_{\underline{\alpha}^{\prime I J K}}^{\bar{\alpha}^{\prime I J K}}, \\
& \left(F_{0}^{I J K}\right)_{x}^{(\text {ends })}=-\beta\left[\frac{\left(2 R+x^{I}\right)\left(1-\cos \alpha^{\prime}\right)}{\kappa r^{I J K 2}}+\frac{\beta \sin \alpha^{\prime}}{\kappa r^{I J K}}\right]_{\underline{\alpha}^{\prime I J K}}^{\bar{\alpha}^{\prime I J K}}, \\
& \left(F_{0}^{I J K}\right)_{y}^{(\text {ends })}=-\beta\left[\frac{\left(1-\cos \alpha^{\prime}\right) y^{J}}{\kappa r^{I J K} 2}\right]_{\underline{\alpha}^{\prime I J K}}^{\bar{\alpha}^{\prime I J K}} .
\end{aligned}
$$

Indices have been left off the $\kappa$ factors.
The length parameter $L$ has been left undetermined so far. There will be a trade-off between numerical stability of the calculation (which favors large $L$ ) and faithful representation (which favors small $L$ ). For the present discussion we assume the true longitudinal beam distribution is Gaussian, with standard deviation $\sigma_{s}$. Three possible prescriptions for the specification of $L$, in order of decreasing $L$, and hence increasingly faithful representation are as follows.

(i) A coarse estimate of space charge effects can be obtained by selecting $L \approx \sqrt{3} \sigma_{s}$ (to match the standard deviation) and simulating only the evolution of transverse coordinates.

(ii) A model for which $L$ is as large as possible without distorting the longitudinal distribution excessively is to represent the longitudinal beam distribution as the convolution of a distribution of reduced (relative to $\sigma_{s}$ ) standard deviation $\sigma_{s}^{\prime}=\sqrt{\sigma_{s}^{2}-L^{2} / 3}$. When convoluted with the uniform distribution corresponding to string length $2 L$, this produces an approximately correct longitudinal distribution having the correct standard deviation. Even a procedure as simple as treating the distribution as the convolution of a uniform distribution of length $2 L^{\prime}$ convoluted with the string length $2 L$ should yield a reasonably valid longitudinal distribution $-L$ and $L^{\prime}$ can be adjusted to give the correct values for the lowest two nonvanishing moments.

(iii) To most faithfully represent the longitudinal distribution, the parameter $L$ can be chosen increasingly small compared to $\sigma_{s}$ until all results are sufficiently unaffected by further reduction.

Another thread, probably more important, concerns emittance dilution due to CSR radiation. The present paper has shown that the self-force-calculated energy lost to CSR agrees with the various far field calculations of Schiff, Nodvick, and Saxon, Schwinger, Tamm, and many more recent authors. Furthermore, in agreement with Saldin et al. at least in the large $\gamma$ region, this energy has been shown to come primarily at the expense of the 
energy of particles near the tail of the bunch. As the bunch is conveyed toward the region where its small emittance is needed it passes through optical elements tuned to focus the beam achromatically. But (barring esoteric rf cavities) there can be nothing in the optics of arcs, chicanes, etc., that treats front particles differently than back particles. So the optics that perfectly focuses, say, the front particles, will not focus the back particles perfectly. The result will be an effective emittance growth of the bunch treated as a whole, at the location where the small emittance is critical to the intended application.

\section{LIMITATIONS AND APPLICATIONS}

The formalism that has been described has various limitations.

Though all formulas given here are fully relativistic (i.e., valid for all $\gamma$ ) they do not account correctly for transitions between regions of different magnetic field since they assume the particle has always been in the same magnetic field. For example, in the fully relativistic regime the forces proportional to $1 / \gamma^{2}$ can presumably be dropped, which implies the absence of space charge effect in drifts. For a particle that has just entered a magnet, the retarded time calculation should segment the effective charge distribution into a straight line segment (just outside the edge) which would not necessarily give a negligible force, and a curved section (just inside). For a bending magnet of length, say, $1 \mathrm{~m}$, and a string length $L$ much less than say, $1 \mathrm{~mm}$, this concern may seem farfetched but, in fact, the relativistic dilation factor makes the problem of magnet entrance (though not magnet exit) quite complicated.

Fortunately the difficulties mentioned in the previous paragraph do not prevent the estimation of emittance growth for some configurations of current interest. The beam line required to bend a short and intense electron beam through an angle that is some substantial fraction of $2 \pi$ is made up mainly of bending magnets. For beam lines like this the assumption that the particles are always in the same magnet field is reasonably good, especially since high precision in the estimation of a coarse parameter (emittance) is not really required.

Another serious complication is the effective reduction of coherent synchrotron radiation due to the "shielding" effect of the conductive beam tube. This effect, first accurately calculated by Schwinger [16], is known to suppress the long wavelength components of CSR. This would tend to reduce emittance growth but, since the fractional energy content at long wavelengths is relatively small, the suppression may be insignificant. Within the string formalism it is straightforward to model the effect of upper and lower vacuum chamber walls by using image strings.

In the string space charge model a crude estimate of the importance of beam-wall effects can be obtained by accounting only for the presence of the inner wall of the vacuum chamber. After finding the tail angle $\underline{\alpha}^{\prime}$, the line joining source point and field point can be checked to see whether it misses the inner chamber wall. If the line misses the chamber wall then the formulas derived so far apply. Otherwise the effective charge distribution is "cut off" at a point determined by a tangency condition, effectively bringing $\underline{\alpha}^{\prime}$ closer to the origin. This (straightforward) calculation has not yet been attempted. An optimist entertains the hope that, as well as accounting for the leading vacuum chamber effect, much of the uncertainty associated with magnet entry will also be cut off by the inner chamber wall.

The various delicate issues that have been mentioned require investigations not yet completed. Also investigations of dependencies on (artificial) string half-length $L$ are required. For these reasons the present paper includes no numerical results.

Currently the formalism is being incorporated into the Unified Accelerator Libraries [18] accelerator simulation framework, so that numerical evaluations can be performed with realistic lattices. For a bunch containing $N$ particles, the number of interbeam forces to be calculated at each bending magnet is $N^{2}$. For multiturn evolution, in the absence of a grid interpolation scheme like that described earlier, computational practicalities would restrict $N$ to be quite small, say 100 . But for a single passage around one circular arc, or fraction thereof, a bunch of, say, 10000 particles can be treated as direct intrabeam scattering of every particle with every other one. Such a calculation is in progress.

\section{APPENDIX: EXPANSIONS FOR RAPID ROOT LOCATION}

In practice it is necessary to solve Eq. (54) numerically but, to correlate with formulas in earlier sections, it is useful to consider closed-form, approximate solutions. Though there seems to be no uniformly appropriate expansion procedure, it seems useful to expand the cosine term for small argument and to treat the $\alpha^{\prime 4}$ term (temporarily) as constant. Defining

$$
\Delta=x^{2}+y^{2}-R^{2}\left(1+\frac{x}{R}\right) \frac{\alpha^{4}}{12},
$$

the resulting (near) quadratic equation for the combination $R \alpha^{\prime}$ becomes

$$
A\left(R \alpha^{\prime}\right)^{2}+B\left(R \alpha^{\prime}\right)+C=0
$$

where

$$
\begin{aligned}
& A=1-\beta^{2}\left(1+\frac{x}{R}\right), \quad B=2 \Delta s, \quad C=\Delta s^{2}-\beta^{2} \Delta, \\
& \frac{B^{2}}{4}-A C=\beta^{2} \Delta s^{2}\left[1+\frac{x}{R}+\frac{\Delta}{\Delta s^{2}}\left(1-\beta^{2}-\beta^{2} \frac{x}{R}\right)\right] .
\end{aligned}
$$


This expansion is inappropriate if $\Delta s \approx 0$-source point and test point close together-in which case nonvanishing $x$ or $y$ invalidates the small $\alpha^{\prime}$ assumption. Since we will only need solutions for source points at the ends of the string, it is only test points near the ends for which approximation (A2) breaks down. By inequality (57) this excludes a relatively insignificant region. The solutions of Eq. (A2) are

$$
R \alpha^{\prime}=\frac{-\Delta s \pm \beta \Delta s \sqrt{1+x / R+\left[1-\beta^{2}(1+x / R)\right] \Delta / \Delta s^{2}}}{1-\beta^{2}(1+x / R)} .
$$

Depending on the sign and magnitude of $x$, the denominator can change sign. This complicates resolving the \pm ambiguity, but, in any case, the correct root has $c t_{r}<0$ negative (and least negative of all negative roots). As a help in picking the correct sign, consider the case $x=$ $y=0$, which yields

$$
\begin{aligned}
R \alpha^{\prime} & \approx \frac{-1 \pm \beta}{1-\beta^{2}} \Delta s \pm \frac{\beta \Delta}{2 \Delta s} \\
& = \begin{cases}-\frac{\Delta s}{1+\beta}+\frac{\beta \Delta}{2 \Delta s}, & \text { upper sign, } \\
-\frac{\Delta s}{1-\beta}-\frac{\beta \Delta}{2 \Delta s}, & \text { lower sign, }\end{cases} \\
c t_{r} & \approx \frac{ \pm 1-\beta}{1-\beta^{2}} \Delta s \pm \frac{\Delta}{2 \Delta s} \\
& = \begin{cases}\frac{\Delta s}{1+\beta}+\frac{\Delta}{2 \Delta s}, & \text { upper sign, } \\
-\frac{\Delta s}{1-\beta}-\frac{\Delta}{2 \Delta s}, & \text { lower sign. }\end{cases}
\end{aligned}
$$

For the source at the front of the string $\Delta s=s_{t}-L<0$. So to get the negative, and hence correct, solution, requires picking the upper sign for the front particle (and the lower sign for the tail particle.) Choosing these signs leads to

$$
\begin{aligned}
c \bar{t}_{r} & =\frac{-L+s_{t}}{1+\beta}+\frac{\bar{\Delta}}{2\left(s_{t}-L\right)}, \\
c \underline{t}_{r} & =-\frac{L+s_{t}}{1-\beta}-\frac{\underline{\Delta}}{2\left(s_{t}+L\right)}, \\
R \bar{\alpha}^{\prime} & =\frac{L-s_{t}}{1+\beta}+\frac{\beta \bar{\Delta}}{2\left(s_{t}-L\right)}, \\
R \underline{\alpha}^{\prime} & =-\frac{L+s_{t}}{1-\beta}-\frac{\beta \underline{\Delta}}{2\left(s_{t}+L\right)} .
\end{aligned}
$$

These solutions correspond to Eqs. (13) and (14). Since the quantities $\bar{\Delta}$ and $\underline{\Delta}$ depend on $\alpha^{\prime}$ it is necessary to evaluate the overall expressions iteratively, first approximating $\alpha^{\prime}$ without these correction terms and then repeating the calculation with them. For $x, y \neq 0$, this procedure fails for small $\Delta s$ (near the string ends) because the correction terms diverge. But for $x=y=0$ the proportionality of $\Delta$ to $\alpha^{\prime 4}$ cancels these divergences. After these substitutions

$$
\begin{aligned}
\bar{\alpha}^{\prime} & \approx \frac{1}{R} \frac{L-s_{t}}{1+\beta}\left(1-\frac{\beta}{24 R^{2}} \frac{\left(L-s_{t}\right)^{2}}{(1+\beta)^{3}}\right), \\
\underline{\alpha}^{\prime} & \approx-\frac{1}{R} \frac{L+s_{t}}{1-\beta}\left(1-\frac{\beta}{24 R^{2}} \frac{\left(L+s_{t}\right)^{2}}{(1-\beta)^{3}}\right) .
\end{aligned}
$$

The correction terms can be regarded as accounting for the orbit curvature. For $x, y \neq 0$ these formulas can be used to resolve signs. By continuity, the upper sign in Eq. (A4) is the appropriate choice of sign for calculating $c \bar{t}_{r}$, at least provided that $x$ is not too positive (so the denominator remains positive.) Similarly the lower sign has to be taken in Eq. (A4) to obtain $c \underline{t}_{r}$.

Even for $x=y=0$ the lower formula of Eqs. (A8) breaks down for $1-\beta$ too small which limits the usefulness of this formula. As an alternative it is practical to solve Eq. (54) numerically to arbitrarily high accuracy. Since the results are needed only as the limits of integrals, numerical values might seem to be sufficient. But a further integration over $s_{t}$ is required if the total force on the string is to be calculated. It seems as if MAPLE is unable to perform this integration with the limits determined only implicitly.

Another approach to solving for the tail of the string is to use a cubic approximation, with its closed-form solution. For $x=y=0$ the cubic approximation to Eq. (56) for $\underline{\alpha}^{\prime}$ is

$$
\underline{\alpha}^{13}+24(1-\beta) \underline{\alpha}^{\prime}+24 \underline{\Delta s} / R=0 .
$$

For these numerical coefficients and for $s_{t}$ in the relevant physical range, this equation has a unique real root. (When the same reduction is performed for $\bar{\alpha}^{\prime}$ there are three real roots and the formulas, while valid, become difficult to use.)

[1] R. Li and Y. Derbenev, Jefferson Laboratory Report No. LJAB-TN-02-054, 2003.

[2] G. Geloni et al., DESY Report No. DESY 03-165, 2003.

[3] A. Piwinski, CERN Report No. CERN 85-43, 1985.

[4] G. Decker, Ph.D. thesis, Cornell University, 1986.

[5] R. Talman, Phys. Rev. Lett. 56, 1429 (1986).

[6] E. Lee, Part. Accel. 25, 241 (1990).

[7] J. Laslett, LBL Report No. PUB-616, Sec. 3, p. 13, 1966.

[8] Y. Derbenev and V. Shiltsev, SLAC Report No. SLACPub-7181, 1996.

[9] D. Bassetti and D. Brandt, CERN Report No. CERN/ LEP-TH/86-04, 1986.

[10] E. Saldin, E. Schneidmiller, and M. Yurkov, DESY Report No. DESY-TESLA-FEL-96-14, 1995; Nucl. Instrum. Methods Phys. Res., Sect. A 417, 158 (1998).

[11] L. Iogansen and M. Rabinovich, Sov. Phys. JETP 35, 708 (1959); 37, 83 (1960); 38, 856 (1960).

[12] A private communication from Vladimar Shiltsev reports that early work by I. E. Tamm, little known even in Russia, preceded most of the known work on coherent synchrotron radiation. 
[13] G. Geloni et al., DESY Report No. 02-048, 2002.

[14] G. Geloni et al., DESY Report No. 03-044, 2003.

[15] O. Jefimenko, Am. J. Phys. 63, 454 (1995).

[16] J. Schwinger, Lawence Berkley Laboratory Report No. LBNL-39088, 1999.
[17] M. Abramowitz and I. Stegun, Handbook of Mathematical Functions (Dover, New York, 1965).

[18] N. Malitsky and R. Talman, BNL Report No. BNL71010-2003, http://www.ual.bnl.gov 